\author{
Universidade de São Paulo \\ Instituto de Física
}

\title{
Modificação do transporte turbulento por polarização eletrostática em plasmas confinados magneticamente
}

\section{Taymara Aline Rodrigues Dias}

Orientador: Prof. Dr. Zwinglio de Oliveira Guimarães Filho

Dissertação de mestrado apresentada ao Instituto de Física da Universidade de São Paulo, como requisito parcial para a obtenção do título de Mestra em Ciências.

Banca Examinadora:

Prof. Dr. Zwinglio de Oliveira Guimarães - Orientador (IFUSP)

Prof. Dr. Gustavo Zampier dos Santos Lima (UFRN)

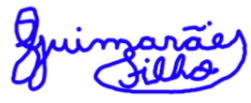

Prof. Dr. Ricardo Luiz Viana (UFPR) 


\section{FICHA CATALOGRÁFICA}

Preparada pelo Serviço de Biblioteca e Informação do Instituto de Física da Universidade de São Paulo

\section{Dias, Taymara Aline Rodrigues}

Modificação do transporte turbulento por polarização eletrostática em plasmas confinados magneticamente. São Paulo, 2021.

Dissertação (Mestrado) - Universidade de São Paulo. Instituto de Física. Depto. de Física Aplicada.

Orientador(a): Prof. Dr. Zwinglio de Oliveira Guimarães Filho

Área de Concentração: Física

Unitermos: 1. Física de Plasmas; 2. Tokamaks; 3. Turbulência Eletrostática. 


\author{
University of São Paulo \\ Physics Institute
}

\title{
Turbulent transport modifications by electrostatic biasing in magnetically confined plasmas
}

\section{Taymara Aline Rodrigues Dias}

Supervisor: Prof. Dr. Zwinglio de Oliveira Guimarães Filho

Dissertation submitted to the Physics Institute of the University of São Paulo in partial fulfillment of the requirements for the degree of Master of Science.

Examining Committee:

Prof. Dr. Zwinglio de Oliveira Guimarães Filho - Supervisor (IFUSP)

Prof. Dr. Gustavo Zampier dos Santos Lima (UFRN)

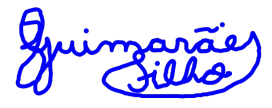

Prof. Dr. Ricardo Luiz Viana (UFPR) 



\section{Agradecimentos}

Ao meu orientador Prof. Dr. Zwinglio de Oliveira Guimarães Filho, por toda a paciência, dedicação e incentivo, sem o qual este trabalho não seria possível.

Aos meus pais, Pedro e Maria, por todo o apoio e carinho.

Às minhas irmãs, Simone, Bianca e Nádia, pelo exemplo e suporte.

Ao Bruno, por todo companheirismo e dedicação.

Ao Dr. Alberto Marcus e ao Prof. Dr. Iberê Luiz Caldas pelas orientações sobre os sistemas Hamiltonianos.

Aos meus colegas de grupo Caike Crepaldi e Martin Zurita pelas conversas sempre frutíferas.

À todos do grupo do TCABR por toda ajuda e oportunidade de utilizar os dados e acompanhar as campanhas.

O presente trabalho foi realizado com apoio da Coordenação de Aperfeiçoamento de Pessoal de Nível Superior - Brasil (CAPES) - Código de Financiamento 001. 


\section{Resumo}

O surgimento de barreiras físicas, sejam espontâneas ou induzidas, ao transporte turbulento de partículas para fora do plasma e a consequente melhora do confinamento global são fenômenos amplamente aceitos e observados em diversas máquinas. Apesar desta universalidade, ainda não existe uma teoria baseada em primeiros princípios que descreva de modo satisfatório as observações experimentais. Neste trabalho estudamos a barreira de transporte turbulento de partículas observada no TCABR, um tokamak de médio porte propício a estudos da região de borda do plasma, sob duas perspectivas distintas, o transporte caótico difusivo devido a deriva $\vec{E} \times \vec{B}$ associado as ondas de deriva e o transporte convectivo de estruturas coerentes de alta densidade geradas na região de borda, aqui referenciadas como "bursts".

Devido a um novo arranjo de sondas disponível no TCABR, simulações numéricas abordando a perspectiva do transporte caótico utilizadas anteriormente pelo grupo, foram revisitados nesta dissertação a partir de valores atualizados de grandezas do plasma e perfis radiais antes inacessíveis.

Por último, abordamos a redução do transporte a partir da óptica dos bursts, investigando o efeito da barreira sobre essas estruturas, seu comportamento médio e como isso reflete nos mecanismos associados à redução do transporte.

Palavras-chave: Tokamak, Barreiras de transporte, Melhora de confinamento, Bursts, Transporte caótico 


\begin{abstract}
The existence and development of physical transport barriers, spontaniously or induced, undermining the particle loss related to turbulence and anomalous transport and its consequential enhanced confinement, are well accepted phenomena observed in many machines. Apart from this universality, a first principles theory that satisfactorily describes the experimental observations is yet to be established, although many are currently being developed comprising distinct approaches. This study engages on two different perspectives to understand the transport barrier phenomenon induced by a biased electrod at the TCABR, a medium sized tokamak ideal to study the plasma edge physics. The first approach takes under review the effect of the bias on the difusive $E \times B$ chaotic transport due to drift waves. The second approach weights the contribution of the transport ralated to convective high density coherent structures, hereby called "burts". A new set of probes allowed revisiting previous studies, focused on the chaotic transport, conducted on the TCABR by the use of a fresh collection of plasma parameters and radial profiles for some of these quantanties that were not originally available. Last, the transport reduction was investigated considerating the impact of the bursts and its consequences for the transport mechanism under review.
\end{abstract}

Keywords: Tokamak, Transport barries, Enhanced confinement, Bursts, Chaotic particle transport 


\section{Lista de Figuras}

1.1 Distribuição da produção mundial de energia elétrica por fonte através do anos. Evidenciando a alta taxa de utilização de combustíveis fosseis, especialmente carvão (coal) e gás natural (gas). (Imagem extraída do projeto "Our World in Data" [3]) . . . . . . . . . . . . . . . . . . . . 10

1.2 Figura extraída da revista [7] de 1981, descrevendo a seção de choque das reações, eixo y, como função da energia, demonstrando a reação de deutério e trítio (D-T) como mais promissora. . . . . . . . . . . . . . .

2.1 Fotografia da visão lateral do Tokamak TCABR, onde é possível ver as bobinas de campo toroidal. (IMAGEM EXTRAÍDA DO BANCO DE IMAGENS DA USP) . . . . . . . . . . . . . . . .

2.2 Representação esquemática da curva característica das sondas eletrostáticas (ou sondas de Langmuir). O $V_{f}$ (potencial flutuante) representa a condição em que o fluxo de elétrons e de íons se anula e as correntes de saturação de íons (elétrons) às condições em que a tensão aplicada à sonda é suficiente para repelir todos os elétrons (íons). Figura extraída da referência [17] . .

2.3 Foto da sonda de 5 pinos usada no Tokamak TCABR para medir em detalhes as propriedades da turbulência em uma mesma posição radial. A posição radial da sonda pode ser alterada entre as descargas de plasma. . . 16

2.4 Foto da sonda de 18 pinos usada para medição simultânea do perfil radial de propriedades do plasma. Foto extraída da referência [17] . . . . . . . .

2.5 Sinais característicos obtidos para a corrente de saturação (figura a) e para o potencial flutuante (figura $b) . \ldots \ldots \ldots$. . . . . . . . .

2.6 Evolução da densidade eletrônica do plasma (a), corrente de plasma (b), tensão aplicada ao eletrodo (c) e a linha $H_{\alpha}$ do Hidrogênio (d), em uma descarga não perturbada do TCABR (curva preta), e uma descarga com a polarização do eletrodo (curva em vermelho) durante $20 \mathrm{~ms}$. A duração total da descarga é cerca de $130 \mathrm{~ms} . \ldots \ldots \ldots$. . . . . . . . .

2.7 Fluxo turbulento de partículas estimado com o uso de uma sonda com arranjo de pinos similar ao da sonda de 5 pinos. O fluxo turbulento antes da polarização do eletrodo é cerca de 3 vezes maior que o observado durante a ação do eletrodo. Figura extraída da referência [13] . . . . . . . . . .

2.8 Relação entre a corrente no eletrodo e a tensão de polarização do eletrodo (gráfico superior) e a razão entre as densidades durante $n_{H}$ e antes $n_{L}$ da polarização do eletrodo em termos da tensão de polarização utilizada (gráfico inferior). Figura extraída da referência [13]. . . . . . . . . . . . 20 
2.9 Espectro de frequências obtidos pela sonda de 18 durante a polarização do eletrodo em $300 \mathrm{~V}$, para posições radiais distintas, da borda do plasma à sombra do limitador. Figura extraída da referência [17] . . . . . . . . .

2.10 Perfil radial da densidade para disparos com polarização do eletrodo, mostrando a posição radial da melhora de confinamento associada à formação da barreira de transporte. Figura extraída da referência [17]. . . . . . . .

2.11 Perfies radiais calculados para o potencial do plasma (a esquerda) e Campo elétrico, sob diferentes tensões aplicadas ao eletrodo. Figura extraída da referência $[17] \ldots \ldots \ldots \ldots \ldots \ldots$

3.1 Representação do sistema de coordenadas na "Slab approximation". Figura extraída de [22]. . . . . . . . . . . . . . . 25

3.2 Mapa estroboscópico para uma onda com valores fixos de $E_{r}$. . . . . . 28

3.3 Mapa estroboscópico para duas ondas com valores fixos de $E_{r} \ldots$. . . . . . 29

3.4 Perfil de campo elétrico para disparo sem eletrodo polarizado (azul) e polarizado em 250V (vermelho). . . . . . . . . . . . . . . . 30

3.5 Mapa estroboscópico para perfil de $E_{r}$ sem polarização de eletrodo . . . . 31

3.6 Mapa estroboscópico para perfil de $E_{r}$ com eletrodo polarizado em $250 \mathrm{~V} \quad 32$

4.1 Exemplo de sinal normalizado de corrente de saturação iônica (proporcional às flutuações de densidade local do plasma) mostrando a presença de picos mais de 2.5 desvios-padrões acima do valor médio, os quais são identificados como bursts. . . . . . . . . . . . . . . .

4.2 Histograma da corrente de saturação iônica mostrando uma assimetria positiva bastante acentuada. . . . . . . . . . . . . . . . 34

4.3 Histogramas de corrente de saturação iônica normalizada obtidos em diversas máquinas de confinamento magnético de plasmas - Tore Supra, Alcator C-MOD e MAST são tokamaks, e PISCES é um dispositivo linear. Figura extraída da referência $[26] \ldots \ldots$. . . . . . . . . .

4.4 Representação esquemática do processo de formação dos bursts por meio da deriva provocada pela curvatura e gradiente do campo magnético. Figura extraída da referência [24]. . . . . . . . . . . . . . . . . 36

4.5 Perfil temporal típico dos bursts observados no tokamak TCABR, obtido pelo uso da técnica de análise condicional. A escala horizontal representa a diferença de tempo em relação ao momento em que os picos identificados como bursts são detectados na sonda. . . . . . . . . . . . . . 37

4.6 Esquema de arranjo dos pinos, cores referentes aos sinais a seguir. . . . . 37

4.7 Média condicional cruzada dos bursts detectados nas três sondas dispostas verticalmente (poloidalmente) na sonda de cinco pinos com a referência para identificação dos tempos condicionados definida pelo pino superior da sonda (curva em azul). As curvas em vermelho e verde correspondem, respectivamente, aos sinais medidos nos pinos localizados $0.5 \mathrm{~cm}$ e $1.0 \mathrm{~cm}$ abaixo do pino de referência. . . . . . . . . . . 38 
4.8 Similar à Figura 4.7, porém, para o caso em que o pino de referência para a média condicional é o pino central da sonda (curva em vermelho). O sinal azul corresponde ao pino superior $(0.5 \mathrm{~cm}$ acima do pino de referência) e a curva verde ao pino inferior $(0.5 \mathrm{~cm}$ abaixo do pino de

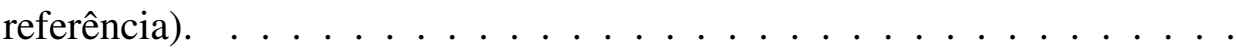

4.9 Similar às Figuras 4.7 e 4.8, porém, para o caso em que o pino de referência para a média condicional é o pino inferior da sonda (curva em verde). O sinal azul corresponde ao pino superior $(1.0 \mathrm{~cm}$ acima do pino de referência) e a curva vermelha ao pino central da sonda $(0.5 \mathrm{~cm}$ acima do pino de referência). . . . . . . . . . . . . . . . .

4.10 Formato condicional dos bursts em diferentes posições radiais na região de borda e sombra do limitador. A escala vertical é a corrente de saturação normalizada e a escala horizontal é o tempo em relação ao momento que os picos identificados como bursts são detectados no sinal de $I_{\text {Sat }}$. . . . .

4.11 Relação entre a velocidade radial $\left(v_{r}=\delta \phi / d \theta\right)$ e a amplitude da perturbação de densidade provocada pelo burst. A linha tracejada em vermelho indica o valor médio da velocidade radial de todos os bursts representados. Velocidades positivas indicam bursts se movendo para fora do plasma. . . .

4.12 Formato condicional dos bursts para um disparo com polarização do eletrodo em $150 \mathrm{~V}$. O sinal azul corresponde ao período antes da polarização do eletrodo, o sinal vermelho ao período em que o eletrodo estava polarizado e o preto ao período pós polarização. . . . . . . . . . . . .

4.13 Formato condicional dos bursts para um disparo com polarização do eletrodo em 200V. O sinal azul corresponde ao período antes da polarização do eletrodo, o sinal vermelho ao período em que o eletrodo estava polarizado e o preto ao período pós polarização. . . . . . . . . . . . .

4.14 Formato condicional dos bursts para um disparo com polarização do eletrodo em $250 \mathrm{~V}$. O sinal azul corresponde ao período antes da polarização do eletrodo, o sinal vermelho ao período em que o eletrodo estava polarizado e o preto ao período pós polarização. . . . . . . . . . . . . .

4.15 Formato condicional dos bursts em diferentes posições radias para um disparo com polarização do eletrodo em $250 \mathrm{~V}$. O sinal azul corresponde ao período antes da polarização do eletrodo, o sinal vermelho ao período em que o eletrodo estava polarizado e o preto ao período pós polarização. 


\section{Lista de Tabelas}

3.1 Parâmetros da simulação que reproduzem as condições típicas da região de borda do tokamak TCABR 


\section{Sumário}

1 Introdução 9

2 Revisão do efeito da polarização de borda no Tokamak TCABR 14

2.1 Sondas eletrostáticas . . . . . . . . . . . . . . . . 15

2.2 Confinamento no TCABR . . . . . . . . . . . . . . . 18

3 Interpretação do efeito do eletrodo pelo modelo de ondas de deriva em plasmas $\quad 23$

3.1 Onda de deriva em Tokamaks . . . . . . . . . . . . . . . . 23

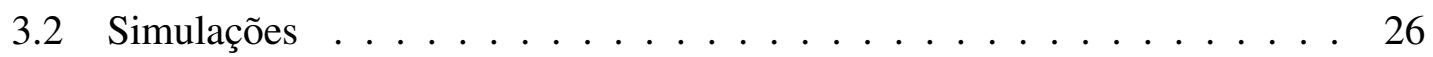

3.3 Ondas de deriva no TCABR $\ldots \ldots \ldots \ldots$

4 Bursts no Tokamak TCABR $\quad 33$

4.1 O processo de propagação convectivo dos bursts . . . . . . . . . . . . 33

4.2 Efeito do eletrodo sobre os Bursts . . . . . . . . . . . . . . . . 42

5 Conclusão 46

Referências $\quad 48$ 


\section{Capítulo 1}

\section{Introdução}

Estima-se que em 2019 62\% da produção mundial de energia elétrica foi oriunda de combustíveis fósseis [1]. Em um cenário mundial que observa um crescimento anual de 1 a $3 \%$ no uso de energia elétrica [2], taxa essa que deve apenas crescer com a popularização de carros elétricos e sistemas modernos de aquecimento, e a ausência de mudanças consideráveis nos níveis de consumo de cada fonte nas últimas décadas 1.1. Pode ser observado na figura 1.1, o consumo de carvão é similar ao de 20 anos atrás e o uso de gás natural vem aumentando.

Apesar do sistema energético mundial ser altamente dependente de combustíveis fosseis é estimado que as reservas mundias desses materiais se esgotem em um prazo de 50 a 100 anos [3], possibilitando uma crise energética global nas próximas décadas.

Esta realidade vem impulsionando o desenvolvimento de novas fontes de energia para suprir a futura demanda. Uma das fontes com o maior potencial de desenvolvimento e implementação comercial é a fusão nuclear, processo que fornece energia às estrelas através da formação de novos núcleos atômicos a partir de outros mais leves, como o hidrogênio e o hélio. Umas das maiores vantagens técnicas da fusão nuclear é a quantidade de energia gerada por volume de matéria prima, razão essa muito maior que a obtida atualmente pelas reações de fissão nuclear, com a vantagem de resíduos químicos mínimos e os riscos de acidentes devido a instabilidades são quase nulos.

Apesar do processo de fusão ser comum em nosso universo, ele tem desafiado a ciência no último século na busca por controlá-lo a ponto de se tornar uma fonte energética de baixo custo, limpa e renovável [4]. O desafio não se encontra no entendimento do processo de fusão, esse é bem definido a décadas, incluindo as reações mais promissoras (Figura 1.2), mas sim o controle e obtenção das condições necessárias para alcançar um processo de fusão em um estado auto sustentável, ignição, onde o processo não mais necessita de fontes externas de energia para realizar as reações.

Para que a fusão nuclear aconteça os nuclídeos devem vencer a força Coulombiana que 


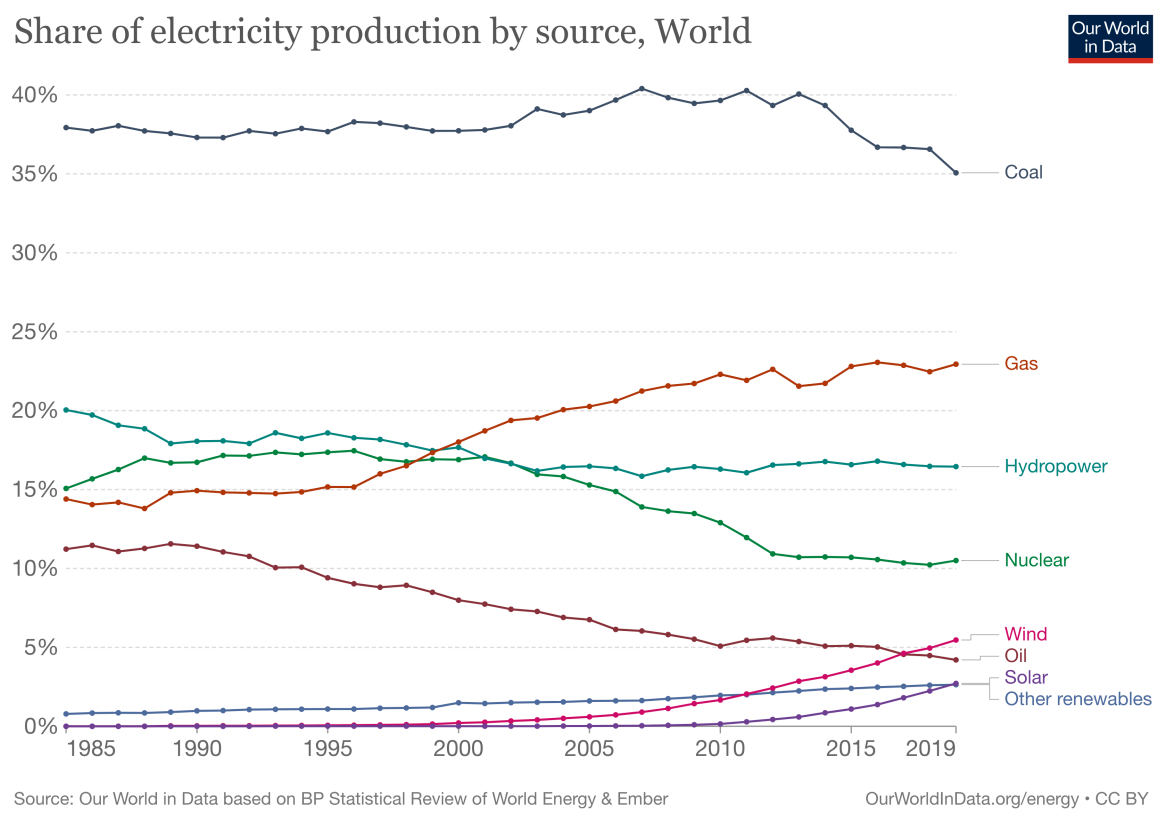

Figura 1.1: Distribuição da produção mundial de energia elétrica por fonte através do anos. Evidenciando a alta taxa de utilização de combustíveis fosseis, especialmente carvão (coal) e gás natural (gas). (Imagem extraída do projeto "Our World in Data" [3])

faz eles se repelirem, tais nuclídeos precisam estar sujeitos a condições de alta temperatura e pressão, e sendo gases candidatos ideais para a fusão devido a alta liberação de energia, sobe estas condições de temperatura e pressão os gases se encontram ionizados e no estado de quase neutralidade de plasma.

Uma das reações de fusão mais promissoras, atualmente em teste e projetada para uso em futuros reatores [5], é a fusão entre deutério e trítio, pois ela possui uma alta seção de choque para uma das menores energias dentre os candidatos à fusão $(20 \mathrm{keV})$, porém com grande liberação de energia, reação representada pela equação a seguir. 1.2[5, 6, 7].

$$
{ }_{1}^{2} \mathrm{D}+{ }_{1}^{3} \mathrm{~T}={ }_{1}^{4} \mathrm{He}+{ }^{0} \mathrm{n}+17,6 \mathrm{MeV}
$$

Para que a energia gerada por fusão seja maior que a gasta para realiza-lá, o plasma deve ter um balanço apropriado entre a densidade de elétrons, o tempo de confinamento e a temperatura iônica, o produto dessas quantidades é um valor utilizado como caracterização da ignição e é conhecido como critério de Lawson [6, 8]. Tal critério estabelece limites mínimos para o produto dessas grandezas.

Um dos métodos de confinar o plasma que vem tendo resultados promissores, é o confinamento magnético em câmaras toroidais, chamadas Tokamaks. Esse método de confinamento usa o fato do plasma ser formado por "partículas carregadas"e portanto agem de acordo com a força de Lorentz quando submetidas a campos eletromagnéticos. 


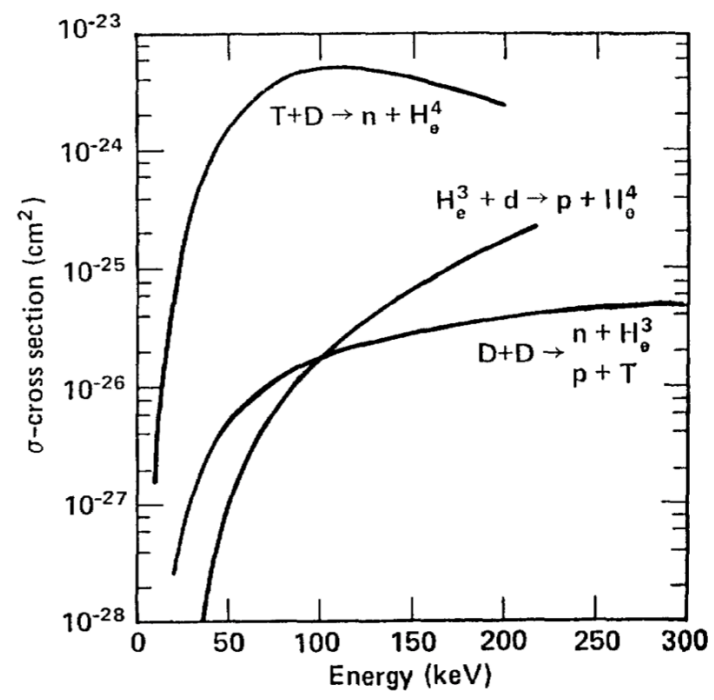

Figura 1.2: Figura extraída da revista [7] de 1981, descrevendo a seção de choque das reações, eixo y, como função da energia, demonstrando a reação de deutério e trítio (D-T) como mais promissora.

Na força de Lorentz, descrita por $\mathbf{F}=q(\mathbf{E}+\mathbf{v} \times \mathbf{B})$, o termo relacionado ao campo magnético faz com que partículas que possuam velocidades paralelas a $\mathbf{B}$ não encontrem resistência enquanto que partículas com velocidades perpendiculares sentem essa força como uma força centrípeta e acabam girando no plano perpendicular à $\mathbf{B}$. Assim é possível confinar as partículas às linhas de campo magnético e o confinamento se torna possível.

Apesar da geometria toroidal dos Tokamaks permitir o confinamento magnético do plasma, ela tem como lado negativo a inomogeneidade do plasma com a presença de gradientes radiais de campo magnético, temperatura, pressão e densidade. Esses gradientes junto com a força colombiana de longo alcance dos íons e elétrons geram um transporte turbulento de partículas para fora do plasma, este processo constante gera a degradação do confinamento, pois carrega para fora da coluna principal energia e partículas, e na presença de eventos mais extremos gera o deposito de material nas paredes da câmara introduzindo impurezas no plasma.

O transporte turbulento e outras instabilidades faziam com que a realização da fusão para uso comercial estivesse muito longe da realidade. Mas em 1982 no antigo Tokamak ASDEX, foi observado um modo espontâneo de confinamento melhorado, onde a densidade e o tempo de confinamento aumentaram drasticamente, simultaneamente com a redução do transporte de energia, partículas e impurezas. Este modo foi chamado de H-Mode (High Mode) e o modo padrão de L-Mode (Low Mode). Nos anos seguintes o H-mode foi reproduzido em outros tokamaks como PDX, o DIII-D e o JET, gerando uma nova onda de suporte aos projetos de Tokamaks [9]. 
Uma dessas iniciativas é o projeto ITER (International Thermonuclear Experimental Reactor), uma parceria mundial na construção de um Tokamak de proporções necessárias para gerar a fusão sem deficit energético, liberação de energia maior que a necessária para gerar a ignição, sendo que atualmente o Tokamak JET detêm o recorde com uma energia gerada por fusão de cerca de $70 \%$ da gasta para aquecer e confinar o plasma [10].

A melhora de confinamento observada no modo-H tem sido relacionada com a formação de uma barreira física ao transporte de partículas localizada na região de borda do plasma, reduzindo a turbulência e o consequente transporte anômalo nessa região. A formação da barreira tem se mostrado altamente dependente do aumento dos gradientes na borda, principalmente ao gradiente do campo elétrico radial.

Foi mostrado que através da criação de campos elétricos radias na região de borda através da polarização de sondas eletrostáticas locais é possível induzir a formação da barreira e a consequente melhora de confinamento esperada $[9,11]$.

A região de borda do plasma parece ser uma peça fundamental no entendimento e controle da melhora de confinamento, porém o fenômeno físico responsável pela barreira ao transporte ainda é uma questão em aberto, tornando a experimentação de borda provenientes de sondas eletrostáticas extremamente relevante.

Graças a universalidade das propriedades da turbulência e as leis de escala $[12,11]$, os estudos realizados em máquinas com densidade e temperaturas menores, em geral de tamanho reduzido, podem ser estendidos a máquinas maiores como o ITER. Isto permite um estudo mais detalhado da região de borda, pois em máquinas de temperaturas menores é possível posicionar sondas na borda do plasma, permitindo medir perfis locais de temperatura, densidade e campo elétrico.

Os estudos presentes nesta dissertação foram conduzidos com dados experimentais do Tokamak TCABR, máquina de pequeno médio porte instalada no IFUSP. Como será discutido no capítulo dois, estudos anteriores mostraram a melhora de confinamento obtida nesta máquina através da polarização de um eletrodo na região de borda [13]. Para interpretar as medidas iniciais desse regime melhorado no TCABR, foi utilizado um modelo teórico onde o transporte de partículas é relacionado ao transporte caótico na direção radial devido a diferença de fase entre a velocidade de fase das ondas de deriva, responsáveis por grande parte do transporte, e a velocidade do fluxo $E \times B$ gerado pelo capo elétrico radial $[14,15,16]$.

Estudos recentes realizados com novas sondas eletrostáticas com diferentes arranjos de pinos, permitiram obter curvas locais de temperatura e portanto dados mais precisos de campo elétrico e densidade. Estes novos dados foram interpretados seguindo um modelo que prevê que a melhora de confinamento é provocada pela dissociação de estruturas coerentes, que viajam para fora do plasma, devido ao cisalhamento do fluxo $E \times B$ na 
direção poloidal gerado pelo gradiente de campo elétrico radial [17].

No capítulo três serão apresentadas as novas simulações realizadas para o modelo de transporte caótico de partículas utilizando dados de potencial do plasma medido por sondas de Langmuir corrigidos pelo perfil de temperatura local obtidos em estudos recentes realizados no TCABR $[17,18]$.

Já no capítulo quatro será estudado o comportamento dos "bursts", estruturas convectivas de alta densidade e temperatura encontradas na região de borda do plasma e relacionadas ao transporte anômalo de energia e partículas, em descargas naturais e sob o efeito da polarização do eletrodo. As alterações provocadas no perfil temporal dos bursts devido à ação do eletrodo são apresentadas neste capítulo.

Finalmente, as conclusões do trabalho são apresentadas no capítulo cinco. 


\section{Capítulo 2}

\section{Revisão do efeito da polarização de borda no Tokamak TCABR}

Atualmente o Instituto de Física da Universidade de São Paulo possui em funcionamento o Tokamak de pequeno médio porte, Tokamak Chauffage Alfvén Brésilien (TCABR), constituído de um raio maior de $R=61,5 \mathrm{~cm}$ e raio menor $r=18 \mathrm{~cm}$. Com um tempo de duração máximo de um disparo com plasma de aproximadamente $110 \mathrm{~ms}$. Seu plasma é caracterizado por um campo magnético toroidal aproximado de $1 T$, corrente de plasma $90 k A$, densidade eletrônica média $\approx 2 \times 10^{-19} \mathrm{~m}^{-3}$, temperatura de elétrons $T_{e}=500 \mathrm{eV}$ e temperatura de íons $200 \mathrm{eV}$ [17].

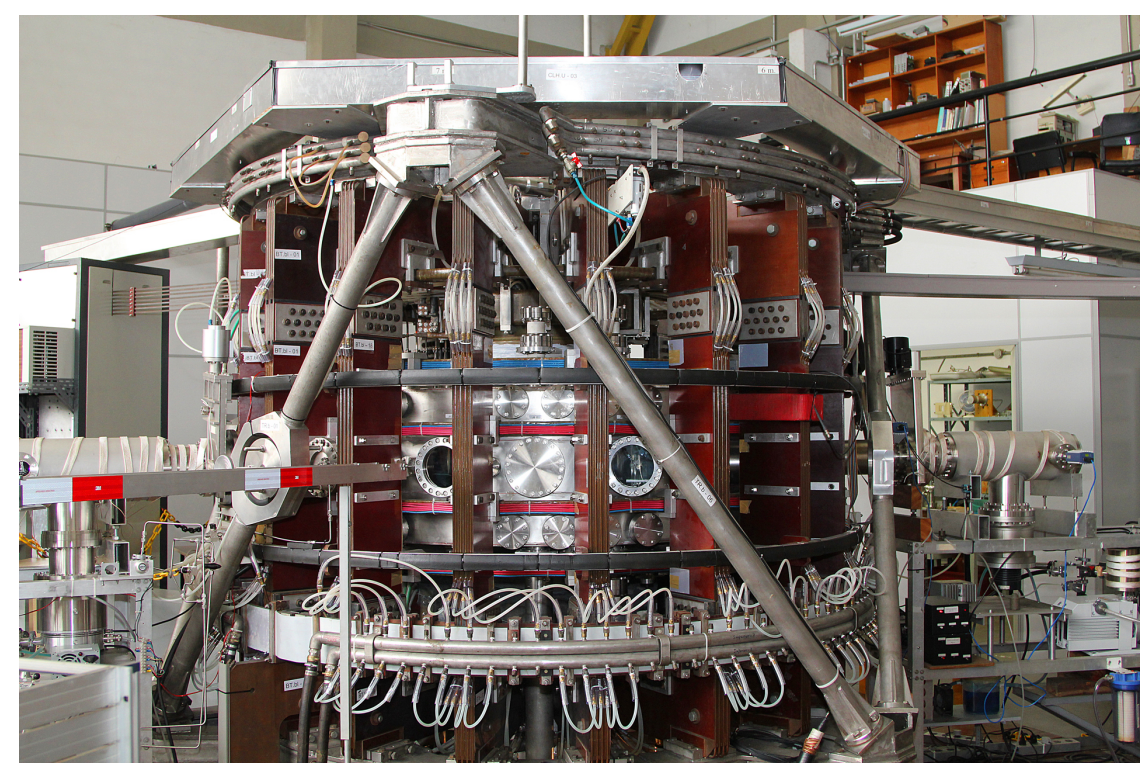

Figura 2.1: Fotografia da visão lateral do Tokamak TCABR, onde é possível ver as bobinas de campo toroidal. (IMAGEM EXTRAÍDA DO BANCO DE IMAGENS DA USP)

O tamanho do TCABR o torna ideal para estudos de turbulência e transporte na região de borda do plasma, pois possibilita a aquisição de dados locais a partir do posicionamento 
de sondas eletrostáticas na borda do plasma.

\subsection{Sondas eletrostáticas}

As sondas eletrostáticas, ou sondas de Langmuir como são conhecidas devido ao nome de seu inventor, o físico Irvin Langmuir, são basicamente eletrodos a serem polarizados com relação a parede da câmara de confinamento. Estas sondas permitem a obtenção de estimativas de temperatura, densidade e potencial do plasma, dependendo do modo em que a sonda é polarizada.

Seu posicionamento na borda do plasma permite a aquisição de dados de forma direta, permitindo um estudo aprofundado da dinâmica da turbulência e transporte nessa região, porém esta forma de aquisição introduz pertubações no plasma que devem ser consideradas [19].

Polarizando positivamente um eletrodo no plasma, coletaremos mais elétrons na sonda, essa corrente de elétrons coletados cresce de acordo com o potencial aplicado até que se atinja o ponto de saturação, onde todos os íons são repelidos e o máximo de elétrons disponíveis é coletado. O efeito contrario também é observado, polarizando negativamente o eletrodo os elétrons são repelidos e pode-se coletar uma corrente de íons, sendo que o ponto de saturação é chamado de corrente de saturação de íons, esta que por sua vez é proporcional a densidade eletrônica na região [20]. A corrente de saturação coletada $\left(I_{s a t}\right)$ pode ser expressa pela equação 2.1 , onde a velocidade dos íons coletados em uma sonda de área A, é aproximada pela velocidade do som para "íons frios" $\left(T_{i}<<T_{e}\right)$.

$$
I_{\text {sat }} \alpha e A n_{e} \sqrt{\frac{k_{B} T_{e}}{m_{i}}}
$$

Onde $k_{B}$ representa a constante de Boltzmann, $T_{e}$ a temperatura eletrônica, $n_{e}$ a densidade eletrônica e $m_{i}$ a massa dos íons.

Entre esses dois pontos existe um ponto de corrente nula, o chamado potencial flutuante. Este potencial de corrente nula esta relacionado ao potencial do plasma ,porém ocorre para uma tensão na sonda bem inferior ao potencial do plasma, $V_{p}$

$$
V_{f}=V_{p}-\gamma \frac{k_{B} T_{e}}{e}
$$

Onde o fator $\gamma$ representa o ajuste local devido a razão entre a corrente de íons a de elétrons.

Assim podemos obter estimativas para grandezas essenciais do plasma como a densidade, temperatura e potencial do plasma $[19,17]$.

Atualmente o TCABR dispõe de duas sondas eletrostáticas de arranjos distintos de 


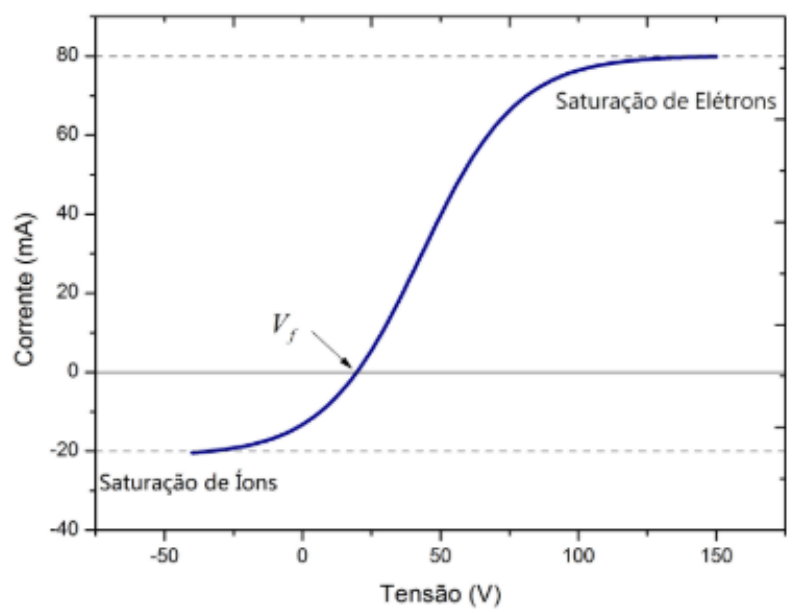

Figura 2.2: Representação esquemática da curva característica das sondas eletrostáticas (ou sondas de Langmuir). O $V_{f}$ (potencial flutuante) representa a condição em que o fluxo de elétrons e de íons se anula e as correntes de saturação de íons (elétrons) às condições em que a tensão aplicada à sonda é suficiente para repelir todos os elétrons (íons). Figura extraída da referência [17]

pinos (eletrodos), uma é formada por um arranjo de cinco pinos em uma mesma posição radial, e o outro arranjo é formado por 18 pinos distribuídos radialmente como pode ser visto nas Figuras 2.3 e 2.4 .

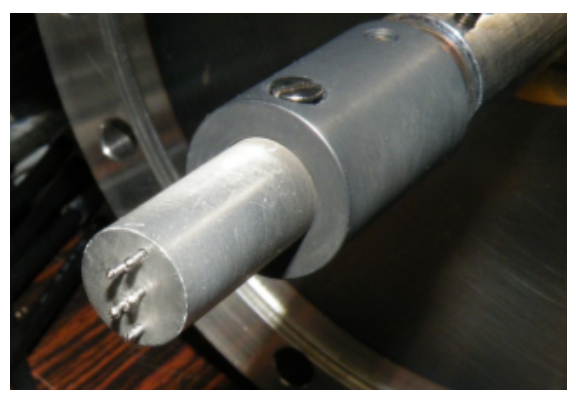

Figura 2.3: Foto da sonda de 5 pinos usada no Tokamak TCABR para medir em detalhes as propriedades da turbulência em uma mesma posição radial. A posição radial da sonda pode ser alterada entre as descargas de plasma.

A sonda de cinco pinos é composta por 5 pinos de tungstênio de $0.5 \mathrm{~cm}$ de comprimento e $0.08 \mathrm{~cm}$ de diâmetro, com pinos espaçados por $0.5 \mathrm{~cm}$ na direção vertical e é utilizada principalmente para o cálculo do transporte de partículas, pois a configuração de pinos espaçados poloidalmente possibilita calcular a velocidade de propagação radial ExB, a partir do campo elétrico poloidal local.

Já a sonda de 18 pinos é composta de 18 pinos de tungstênio de $0.3 \mathrm{~cm}$ de comprimento e $0.08 \mathrm{~cm}$ de diâmetro com cada fileira de pinos distantes $0.5 \mathrm{~cm}$ na direção radial. Seu arranjo radial favorece o estudo de perfis radias das grandezas medidas, tornando-se 


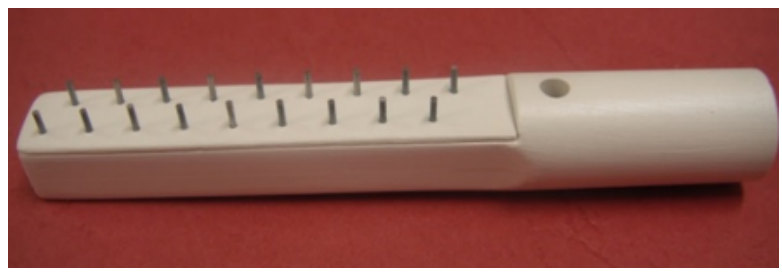

Figura 2.4: Foto da sonda de 18 pinos usada para medição simultânea do perfil radial de propriedades do plasma. Foto extraída da referência [17]

extremamente pertinente no estudo da região de formação da barreira de transporte.

Na figura 2.5 temos os sinais característicos obtidos para a corrente de saturação de íons (a) e o potencial flutuante (b) a partir das sondas de Langmuir.
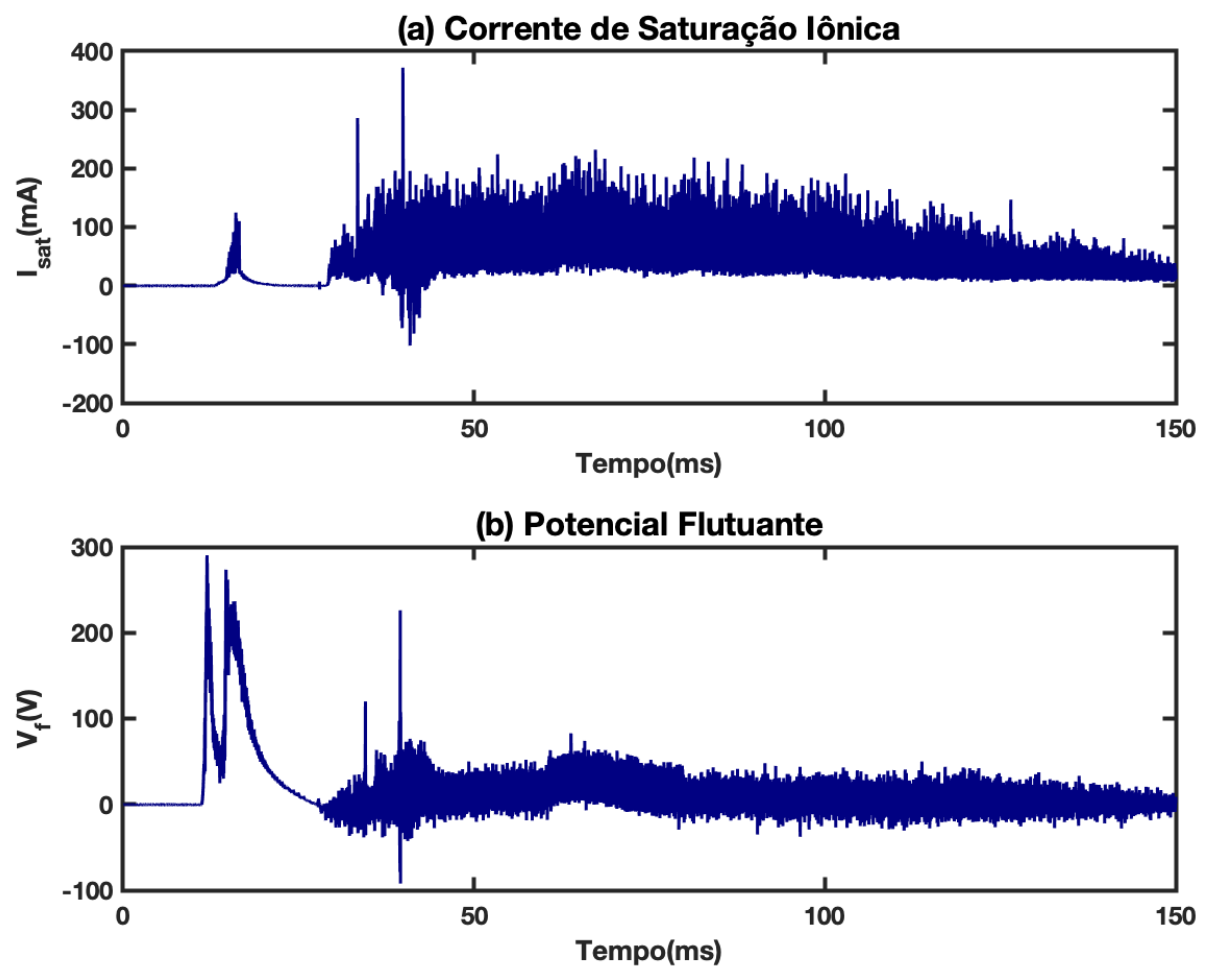

Figura 2.5: Sinais característicos obtidos para a corrente de saturação (figura a) e para o potencial flutuante (figura $b$ ).

Essas medidas foram utilizadas para a obtenção do parâmetros locais da borda do plasma, perfis de temperatura, campo elétrico e turbulência, utilizados nas simulações discutidas no capitulo 3. E para a caracterização e análise dos burts (capitulo 4). 


\subsection{Confinamento no TCABR}

No TCABR o regime de confinamento melhorado é induzido pela polarização de um eletrodo na borda do plasma mantida um determinado período enquanto o plasma se encontra estável (região de platô no caso de uma descarga não perturbada, como representado na Figura 2.6.). A seguir disparos característicos na presença do eletrodo polarizado e sem a polarização são descritos.
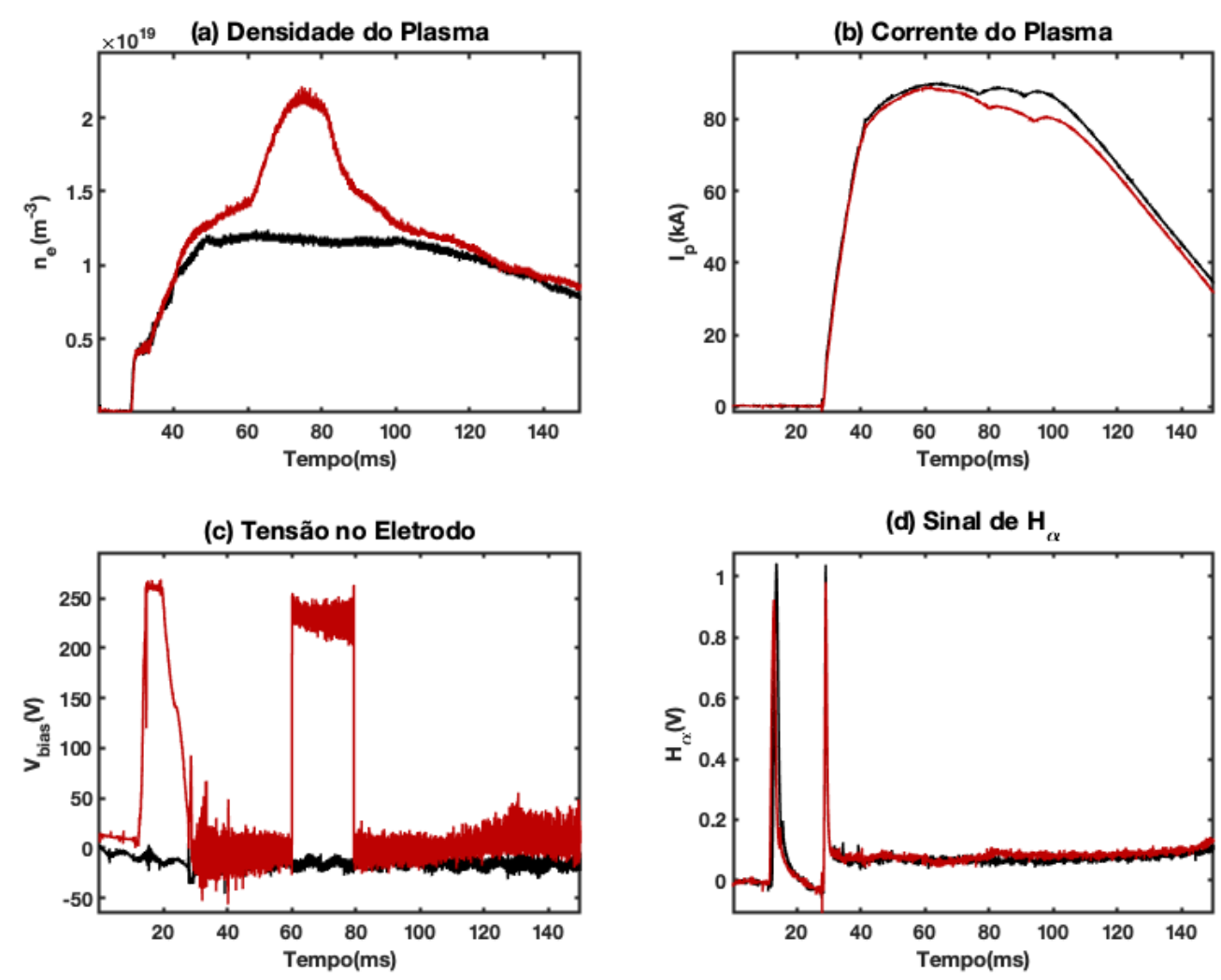

Figura 2.6: Evolução da densidade eletrônica do plasma (a), corrente de plasma (b), tensão aplicada ao eletrodo (c) e a linha $H_{\alpha}$ do Hidrogênio (d), em uma descarga não perturbada do TCABR (curva preta), e uma descarga com a polarização do eletrodo (curva em vermelho) durante $20 \mathrm{~ms}$. A duração total da descarga é cerca de $130 \mathrm{~ms}$.

O aumento de densidade do plasma observado na curva vermelha no quadro (a) da Figura 2.6, também é obtido no TCABR e em outras máquinas através da inserção de mais matéria na câmara, gás puff. Apesar do aumento da densidade esse fenômeno não representa uma real melhora de confinamento, assim estudos anteriores buscaram estudar a melhora de confinamento em função da tensão aplicada ao eletrodo [13].

Esses estudos mostraram que a melhora de confinamento no centro do plasma do TCABR é, como observado em outras maquinas, acompanhado da diminuição do trans- 
porte turbulento de partículas na região de borda, indicando a aparição da barreira, como pode ser visto no quadro (a) da Figura 2.6, que mostra o aumento da densidade do plasma devido à polarização do eletrodo (curva em vermelho no quadro (c)). Esse aumento de densidade ocorre lentamente, com a densidade aumentando durante os primeiros $10 \mathrm{~ms}$ após a aplicação do eletrodo.

A Figura 2.7 mostra a redução do fluxo turbulento de partículas obtido pela aplicação do eletrodo.

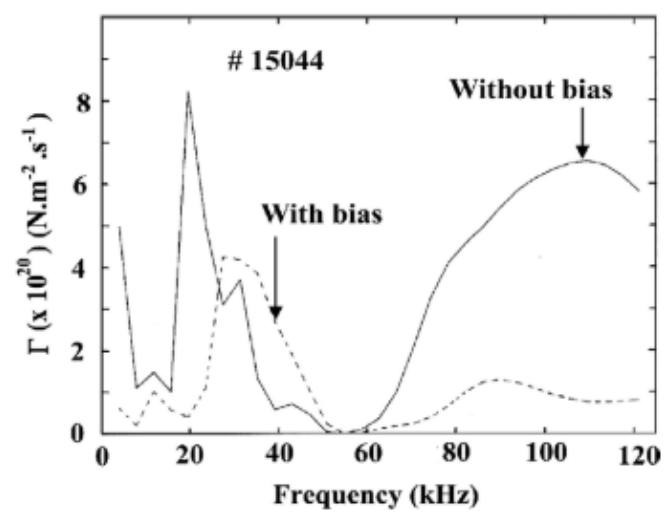

Figure 11. Frequency transport spectra.

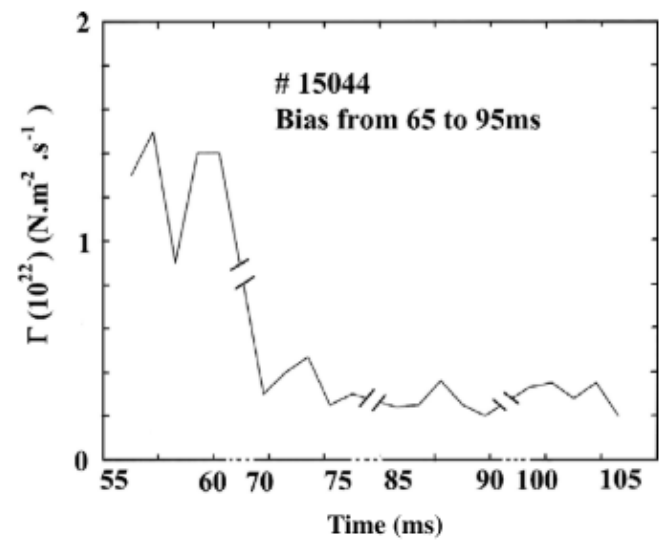

Figura 2.7: Fluxo turbulento de partículas estimado com o uso de uma sonda com arranjo de pinos similar ao da sonda de 5 pinos. O fluxo turbulento antes da polarização do eletrodo é cerca de 3 vezes maior que o observado durante a ação do eletrodo. Figura extraída da referência [13]

Ao estudar a dependência entre a melhora de confinamento e a tensão aplicada ao eletrodo [13], observou-se que o efeito de melhora do confinamento só ocorre para potenciais positivos e parece ter um limiar de cerca de 200 V (ver Figura 2.8). 


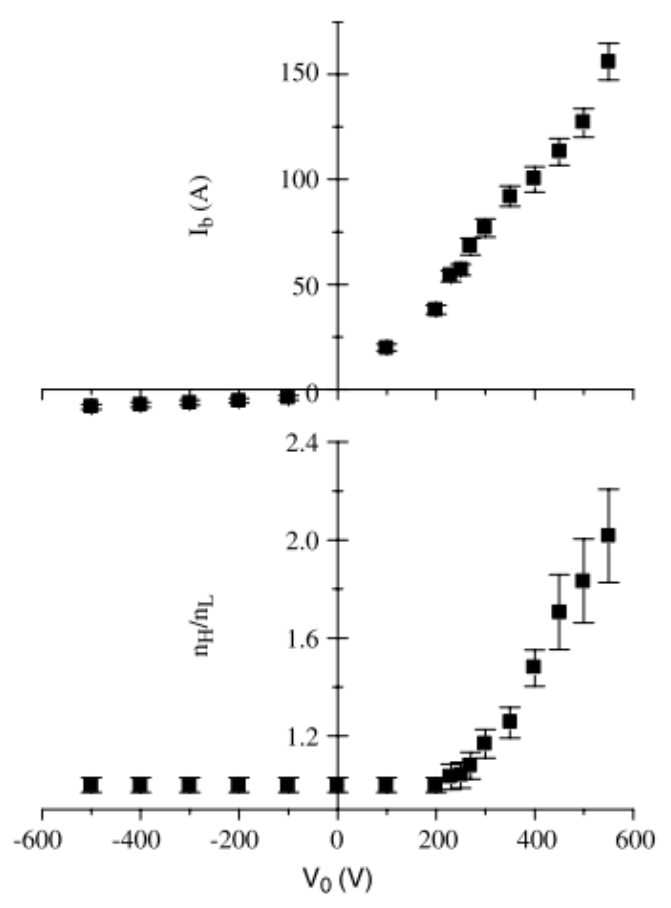

Figura 2.8: Relação entre a corrente no eletrodo e a tensão de polarização do eletrodo (gráfico superior) e a razão entre as densidades durante $n_{H}$ e antes $n_{L}$ da polarização do eletrodo em termos da tensão de polarização utilizada (gráfico inferior). Figura extraída da referência [13].

Em 2007 estes resultados foram interpretados sobe a óptica do transporte caótico de partículas devido a ressonância entre a velocidade de fase das ondas de deriva e a velocidade de deriva $E \times B$. Os parâmetros utilizados nas simulações realizadas em 2007 foram estimados, a partir dos dados coletados com uma sonda de arranjo similar à sonda de 5 pinos (uma sonda com 6 pinos e duas colunas, uma com 4 e outra com 2 pinos). Foram utilizados valores médios de amplitude, frequência, número de onda poloidal da turbulência e o valor médio do campo elétrico radial na região de borda. Foi mostrado que a alteração do campo elétrico observada experimentalmente, faz com que a região de borda que inicialmente é uma região propicia ao transporte radial, passe a ter o movimento das partículas confinadas na região, o que justificaria a diminuição do transporte radial de partículas nessa região na presença do eletrodo polarizado.

Recentemente foi confeccionada uma sonda de alcance radial, a sonda de 18 pinos, permitindo estudos mais detalhados e precisos da região de borda. Utilizando essa sonda foi possível mapear radialmente a melhora de confinamento e estimar o posicionamento da barreira [17]. Com essa nova sonda, o perfil radial da redução da turbulência durante o período de atuação do eletrodo pode ser identificado (ver Figura 2.9). 


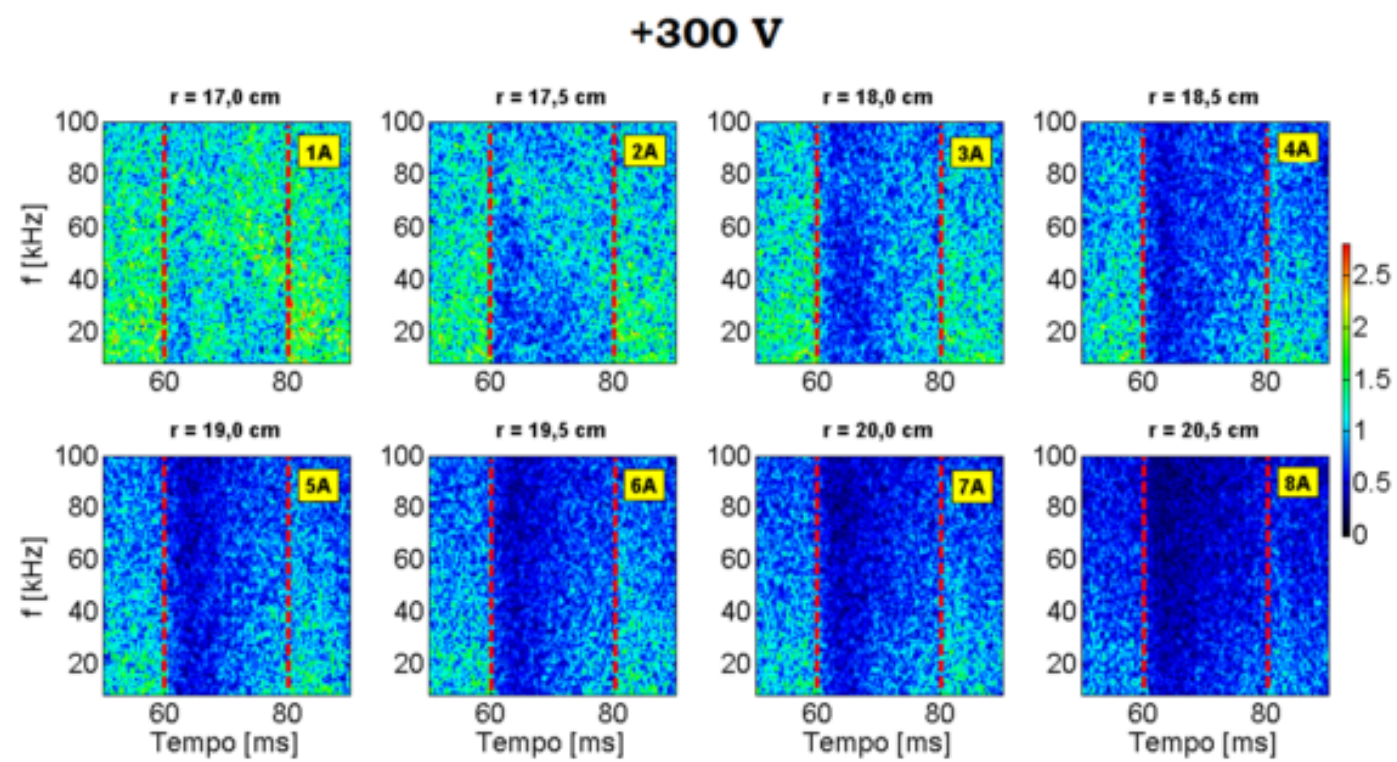

Figura 2.9: Espectro de frequências obtidos pela sonda de 18 durante a polarização do eletrodo em $300 \mathrm{~V}$, para posições radiais distintas, da borda do plasma à sombra do limitador. Figura extraída da referência [17]

Esses resultados detalham a transição do modo L para o modo H no TCABR, exaltando a redução da turbulência sob a ação do eletrodo. Evidências do surgimento de uma barreira de transporte na região próxima à $17,5 \mathrm{~cm}$ pode ser vista na Figura 2.10, que mostra que como resultado da polarização do eletrodo a densidade aumenta nas posições radiais mais internas (até $17 \mathrm{~cm}$ ) ao passo que diminui nas mais externas (após $18 \mathrm{~cm}$ ).

Estes resultados de melhora de confinamento foram interpretados à luz de um modelo que prevê que a diminuição do transporte radial é devido à decorrelação, "quebra", de estruturas coerentes, transportadoras de energia e partículas para fora do plasma. Essas quebra é devido ao cisalhamento da velocidade do fluxo poloidal gerado pelo gradiente do campo elétrico radial, esse que aumenta com o aumento da tensão aplicada no eletrodo, como mostrado na Figura 2.11. Os resultados experimentais foram compatíveis com a previsão teórica de regime de decorrelação das estruturas coerentes [17, 18].

Nos capítulos a seguir ambos os modelos, transporte caótico e decorrelação serão abordados. 

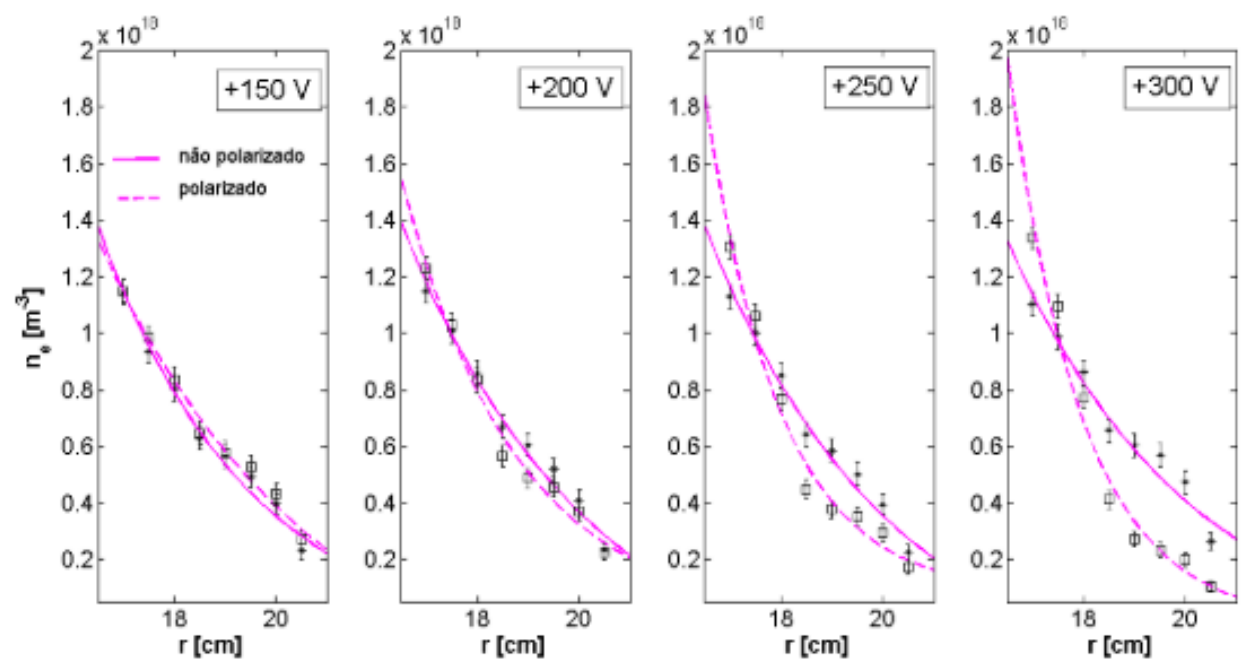

Figura 2.10: Perfil radial da densidade para disparos com polarização do eletrodo, mostrando a posição radial da melhora de confinamento associada à formação da barreira de transporte. Figura extraída da referência [17].
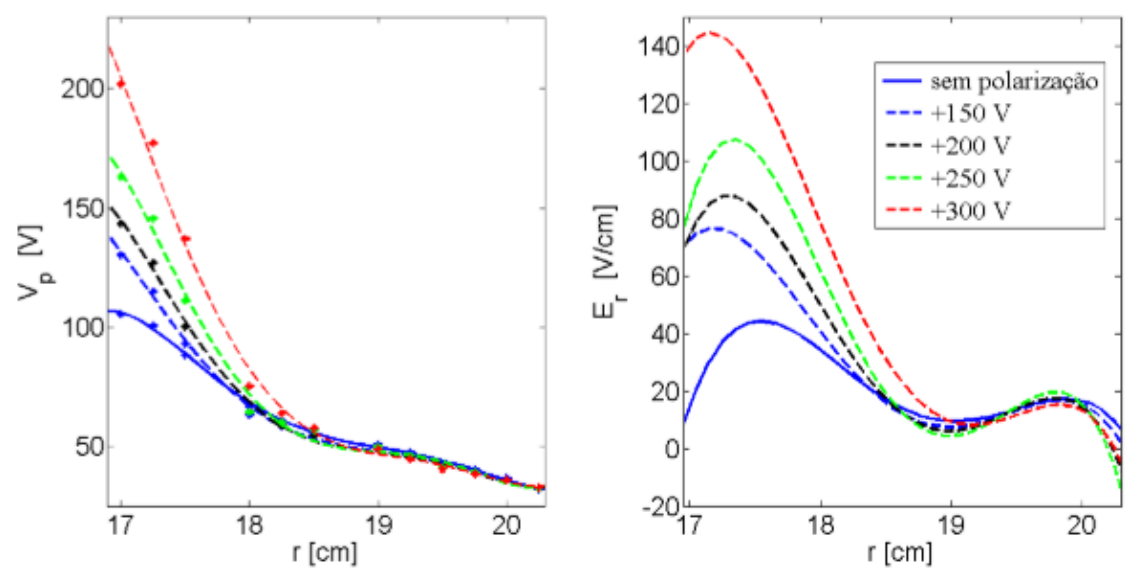

Figura 2.11: Perfies radiais calculados para o potencial do plasma (a esquerda) e Campo elétrico, sob diferentes tensões aplicadas ao eletrodo. Figura extraída da referência [17] 


\section{Capítulo 3}

\section{Interpretação do efeito do eletrodo pelo modelo de ondas de deriva em plasmas}

\subsection{Onda de deriva em Tokamaks}

O entendimento e controle do transporte de partículas em plasmas magneticamente confinados tem sido considerado uma das tarefas mais importantes nos estudos de confinamentos toroidais [21]. Uma das teorias iniciais mais bem sucedidas para o transporte de partículas é a chamada teoria neoclássica de transporte, que adiciona a teoria de transporte de gases elementos do plasma e do confinamento toroidal: a ação da força Coulombiana de longo alcance nas colisões e a ação do campo magnético inomogêneo inerente da geometria de confinamento. Porém o coeficiente de transporte obtido pelo tratamento neoclássico ainda não é suficiente para explicar os altos níveis de transporte observados experimentalmente. A explicações aceita para este fenômeno se baseia no comportamento coletivo das partículas, gerado pela força Coulombiana, que faz com que as partículas se organizem em estruturas coerentes como ondas. A evolução dessas ondas, através de mecanismos diversos, pode levar o plasma a um estado turbulento onde o transporte é favorecido [22].

Acredita-se que grande parte do transporte radial de partículas no plasma é devido à turbulência associada as ondas de deriva de baixa frequência $\left(\omega_{d w}\right)$, inferiores a frequência ciclotrônica dos íons, $\omega_{d w}<\omega_{c i}$ :

$$
\omega_{c i}=\frac{e B}{m_{i} c}
$$

Onde $m_{i}$ é a massa dos íons e $B$ o campo magnético.

As ondas de deriva são originarias da deriva $E \times B$ através do campo magnético das correntes provenientes dos gradientes de temperatura e densidade presentes no plasma [23]. 
Uma boa descrição do surgimento das ondas de deriva é dada pela descrição macroscópica do plasma através da dinâmica de dois fluidos, um representando os íons e o outro o os elétrons, acopladas às equações de Maxwell. Para obter uma solução analítica para essas equações de movimento dada uma pequena variação na condição de equilíbrio, é necessário impor algumas restrições físicas. Um dos tratamentos mais utilizados para ondas de deriva em Tokamaks considera as seguintes condições:

- $T_{i}<<T_{e}$ o plasma é considerado de íons frios;

- O efeito de colisões no transporte é muito pequena, tal que os elétrons estão em equilíbrio local em uma distribuição Maxwelliana: $n=n_{0} \exp \left(-\frac{e \delta \phi}{T_{0 e}}\right)$;

- Quaseneutralidade local $n_{e} \approx n_{i}$.

Com essas aproximações obtêm-se a equação de movimento não linear HasegawaMima, ela descreve a evolução de ondas no plasma geradas por inomogeneidades, como gradientes de temperatura e densidade. Em sua forma normalizada [14, 22], para baixas frequências $(k H z)$, ela é expressa por:

$$
\frac{\partial\left(\nabla^{2} \phi-\phi\right)}{\partial t}-\left[\left(\boldsymbol{\nabla} \phi \times \hat{e}_{z} \cdot \boldsymbol{\nabla}\right]\left[\nabla^{2} \phi-\ln \left(\frac{n_{0}}{\omega_{c i}}\right)\right]=0\right.
$$

Onde phi representa o potencial eletrostático e $n_{0}$ a densidade local.

Considerando uma perturbação de densidade, nos temos a seguinte equação de dispersão [22]:

$$
\omega_{k}=-\frac{1}{1+k_{\perp}^{2}}\left[\left(\bar{k} \times \hat{e}_{z}\right) \cdot \nabla \ln \left(\frac{n_{0}}{\omega_{c i}}\right)\right]
$$

As ondas encontradas como solução dessa equação tem o formato [14, 22]:

$$
\phi=A \sin \left(k_{x} x\right) \cos \left(k_{y} y-\omega_{t}\right)
$$

Onde as coordenadas são a partir da aproximação local, "slab approximation"(ver Figura 3.1), para uma superfície toroidal, onde as coordenas cartesianas são aproximadas às coordenadas cilíndricas. A direção do campo magnético toroidal principal (aqui considerado constante) é representada pela direção $\hat{z}$, a direção radial é descrita pela coordenada $\hat{x}$ e a direção poloidal por $\hat{y}$.

Assim podemos descrever matematicamente o potencial eletrostático na presença dessas ondas como um valor de equilíbrio e um termo devido a essas flutuações que caminhe na direção poloidal e uma amplitude radial modulada devido as observações experimentais de uma modulação radial da amplitude do campo elétrico tal que: 


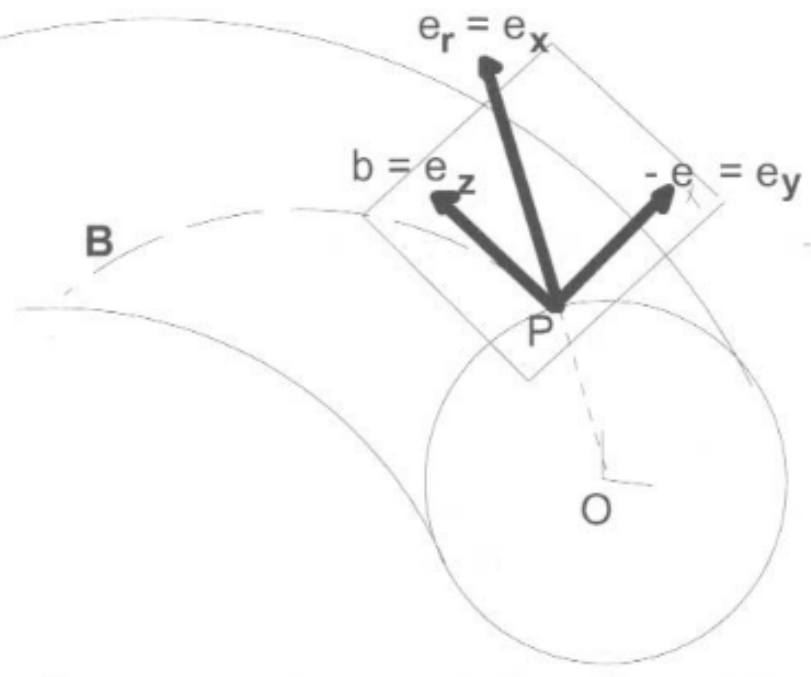

Figura 3.1: Representação do sistema de coordenadas na "Slab approximation". Figura extraída de [22].

$$
\phi(r)=\phi_{0}(r)+\sum_{i=0}^{N} A_{i} \sin \left(k_{r i} r\right) \cos \left(k_{\theta i} \theta-w_{i} t\right)
$$

Onde $k_{r}$ e $k_{\theta}$ são respectivamente os números de onda radias e poloidais.

Assim podemos estimar o transporte $E \times B$ de partículas associado à essas ondas de deriva:

$$
\mathbf{v}_{\mathbf{E} \times \mathbf{B}}=\frac{\vec{E} \times \vec{B}}{B^{2}}
$$

Dado o campo magnético homogêneo e constante na direção toroidal ( $\hat{z}$, e o campo elétrico radial dado por $\vec{E}=-\nabla \phi(r, \theta, t)$ a velocidade de deriva $E \times B$ dos centros guias das partículas é dado por:

$$
\begin{aligned}
\mathbf{v}_{\mathbf{E} \times \mathbf{B}}= & \frac{1}{B_{0}}\left[\sum_{i}^{N} \frac{A_{i} k_{\theta_{i}}}{r} \sin \left(k_{r_{i}} r\right) \sin \left(k_{\theta_{i}} \theta-\omega_{i} t\right) \hat{r}\right. \\
& \left.+\left(\frac{\delta \phi_{0}(r)}{\delta r}+\sum_{i}^{N} A_{i} k_{r} \cos \left(k_{r_{i}} r\right) \cos \left(k_{\theta_{i}} \theta-\omega_{i} t\right)\right) \hat{\theta}\right]
\end{aligned}
$$

Tal que: 


$$
\begin{aligned}
& \frac{\partial r}{\partial t}=\frac{1}{R B_{0}} \sum_{i}^{N} A_{i} k_{\theta_{i}} \sin \left(k_{r_{i}} r\right) \sin \left(k_{\theta_{i}} \theta-\omega_{i} t\right) \\
& \frac{\partial \theta}{\partial t}=\frac{1}{B_{0}} \frac{\delta \phi_{0}(r)}{\delta r}+\frac{1}{B_{0}} \sum_{i}^{N} A_{i} k_{r} \cos \left(k_{r_{i}} r\right) \cos \left(k_{\theta_{i}} \theta-\omega_{i} t\right)
\end{aligned}
$$

Para uma onda este sistema de equações é integrável e possui uma descrição hamiltoniana que permite isolar e reduzir os parâmetros do sistema em um parâmetro de controle $U[14]$ :

$$
U=\frac{B_{0}}{A_{1} k_{r 1}}\left[\frac{1}{B_{0}} \frac{\delta \phi_{0}(r)}{\delta r}-\frac{\omega_{1}}{k_{\theta 1}}\right]
$$

Este parâmetro de confinamento representa a relação entre a velocidade de deriva $E \times B$ dos centros guias e a velocidade de fase da onda de deriva.

\subsection{Simulações}

Para analisar o transporte radial de partículas devido a essa dinâmica, as equações de movimento 3.8 foram integradas numericamente.

As rotinas necessárias foram desenvolvidas em Matlab, onde o integrador numérico ode45 do tipo Runge-Kutta de quarta ordem foi utilizado.

As rotinas desenvolvidas permitem que todos os parâmetros sejam funções do raio $r$, reproduzindo os perfis locais observados no TCABR.

Os parâmetros utilizados foram estimados a partir dos dados experimentais provenientes das sondas de Langmuir.

A partir dos dados da sonda de cinco pinos podem ser obtidos valores médios do número de onda poloidal $k_{\theta}$ da turbulência na região de borda, assim como a velocidade de fase e amplitude das ondas nessa região.

Já a sonda de 18 pinos nos permite obter um perfil radial do campo elétrico, corrigido pela temperatura local, e uma estimativa do número de onda radial $k_{r}$.

Para realizar a integração, os valores de $B_{0}, r, A_{0}, k_{r}, k_{\theta}$ e $\omega$ foram estimados a partir de dados provenientes do TCABR para disparos com e sem eletrodo.

Diferentemente dos estudos realizados anteriormente [14], foi possível, graças ao novo arranjo de sondas radiais, estimar experimentalmente o valor de $k_{r}$ e obter o potencial do plasma de forma mais precisa dada a contribuição da temperatura na região.

Os valores utilizados na integração foram normalizados (U.E. na tabela 3.1) para facilitar o tratamento e evitar erros numéricos durante a integração.

$\mathrm{O}$ estudo foi concentrado na região de borda do plasma $r>17.0 \mathrm{~cm}(r>0.94$, 


\begin{tabular}{|c||c|c|}
\hline Parâmetros & SI & U.E. \\
\hline$R$ & $0.2 m$ & $1 d$ \\
$B_{0}$ & $1 T$ & $0.1 b$ \\
$\omega$ & $5.10^{4} s^{-1}$ & $5 t^{-1}$ \\
$k_{r}$ & $1.10^{3} m^{-1}$ & $200 d^{-1}$ \\
$k_{\theta}$ & $20 m^{-1}$ & $4 d^{-1}$ \\
$A$ & $8.10^{-4} V m^{-1}$ & $4.10^{-4} v d^{-1}$ \\
\hline
\end{tabular}

Tabela 3.1: Parâmetros da simulação que reproduzem as condições típicas da região de borda do tokamak TCABR

normalizado para $r / a$, com $a=18.0 \mathrm{~cm}$ ) sendo que a região confinada do plasma termina em $r=a=18.0 \mathrm{~cm}(r=1.0)$.

Escolhendo valores fixos de campo elétrico radial $\frac{\delta \phi_{0}(r)}{\delta r}=E_{r}$ e considerando apenas uma onda tal que o parâmetro de confinamento U tenha um valor fixo entre zero e 1.5, observamos que o sistema possui essencialmente órbitas confinadas em formatos de células como na Figura 3.2a, e órbitas abertas, representadas por linhas na Figura 3.2b. 


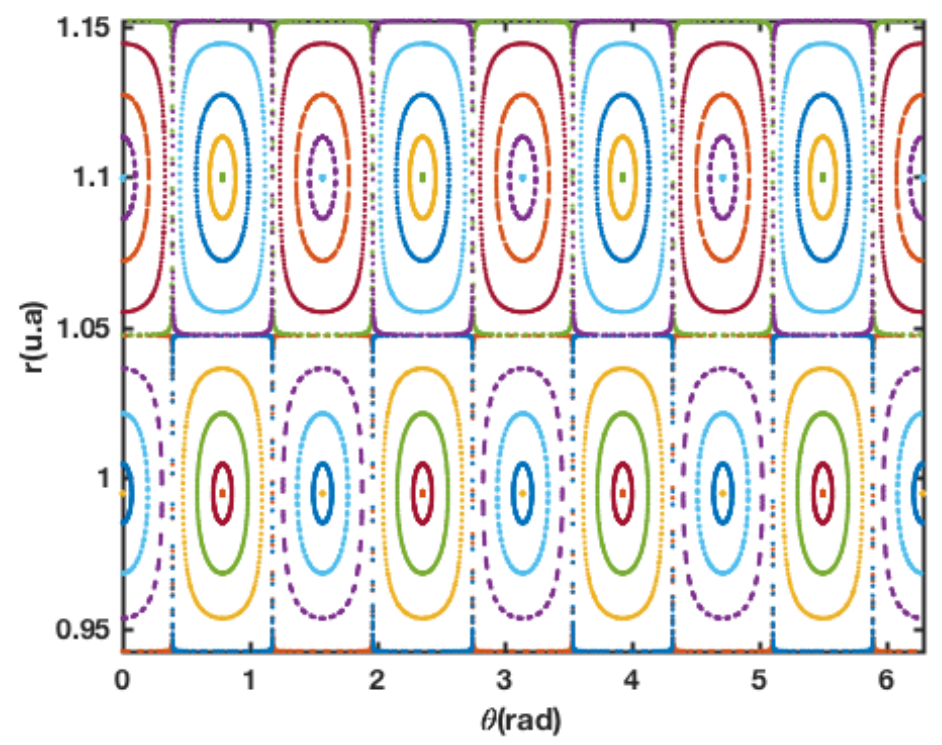

(a) $\mathrm{U}=0$

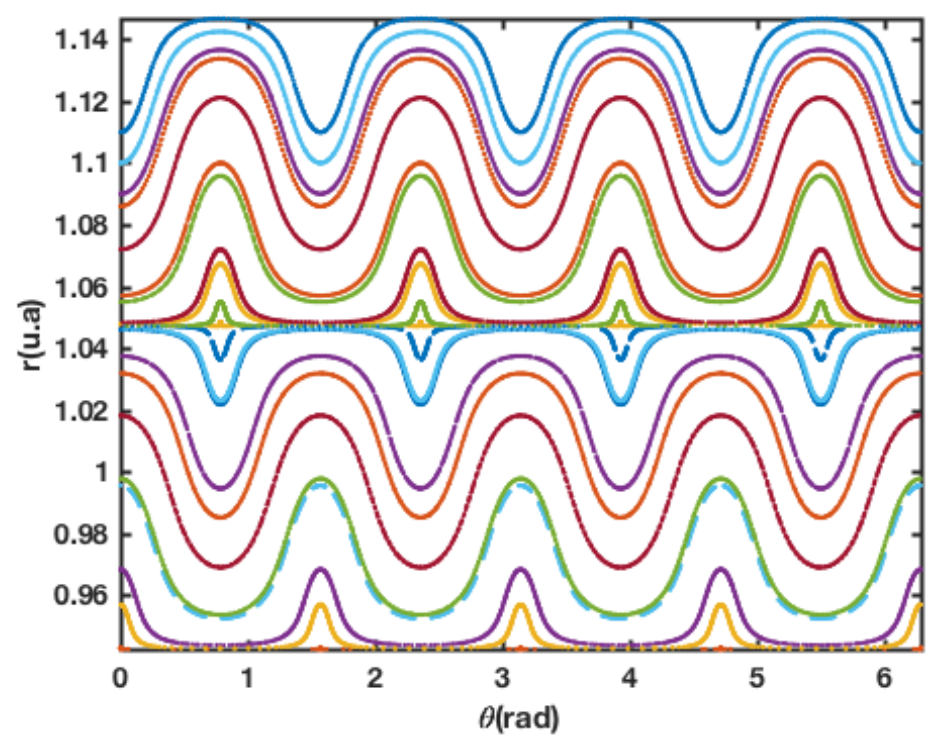

(b) $\mathrm{U}=1.5$

Figura 3.2: Mapa estroboscópico para uma onda com valores fixos de $E_{r}$.

Para $U=0$ temos a ressonância entre a velocidade de fase das ondas e a velocidade de deriva $E \times B$ dos centros guias. Como pode ser visto na Figura 3.2a para $U=0$ existem apenas órbitas fechadas, essas orbitas fechadas na presença de uma segunda onda, que torna o sistema caótico, geram trajetórias que percorrem longas distâncias na direção radial, como pode ser visto na Figura 3.3a, onde uma segunda onda é adicionada. Já as linhas abertas apresentadas na Figura 3.3b, não permitem essa grande propagação radial. 


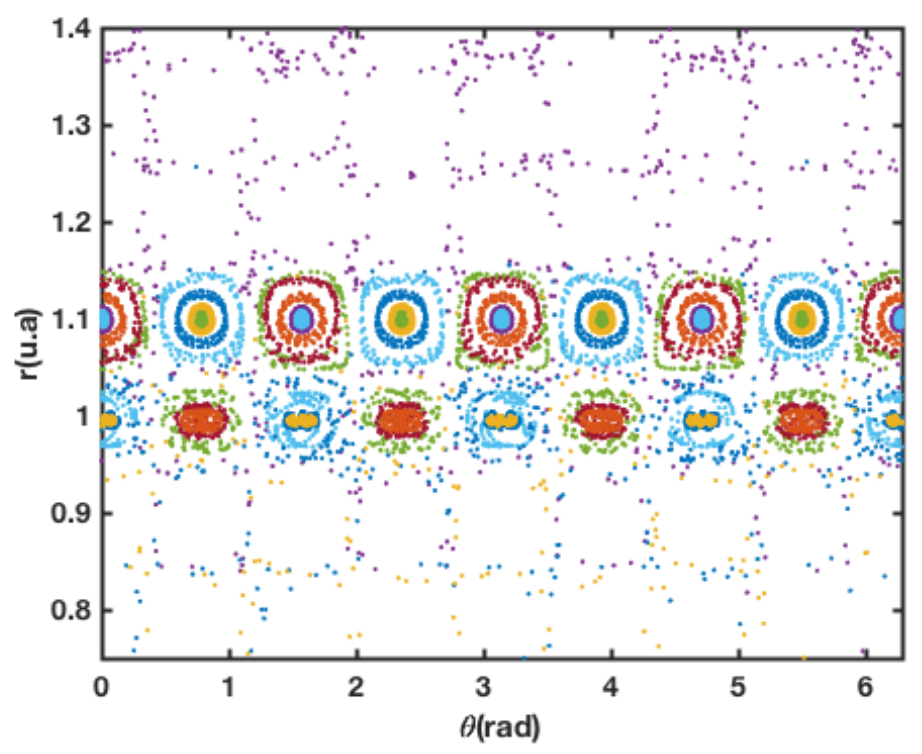

(a) $\mathrm{U}=0$

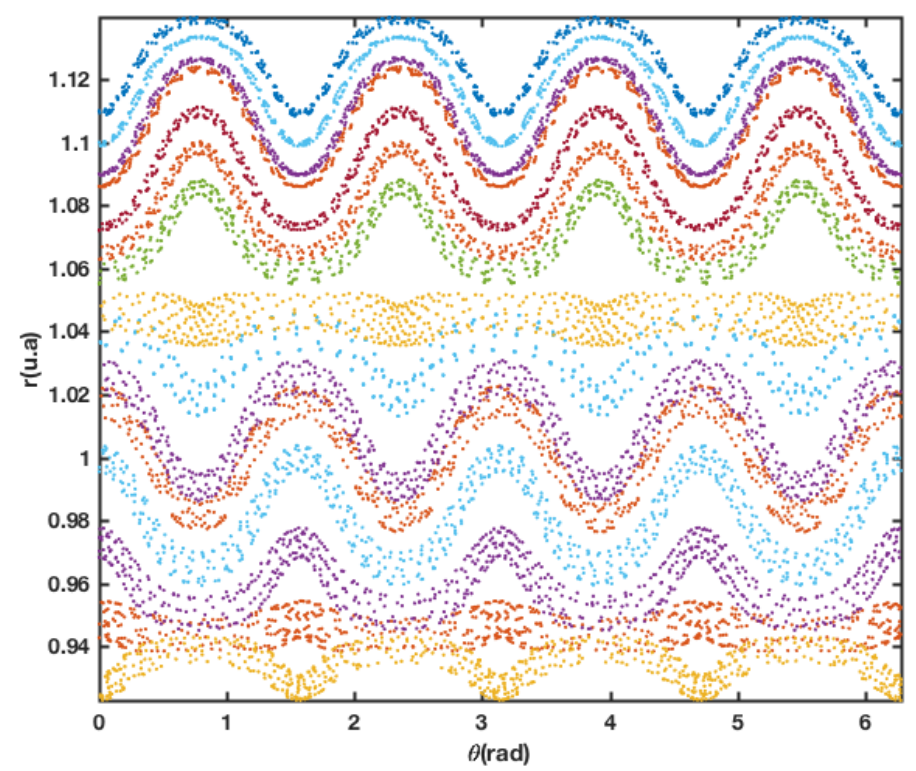

(b) $\mathrm{U}=1.5$

Figura 3.3: Mapa estroboscópico para duas ondas com valores fixos de $E_{r}$.

Para valores fixos do campo elétrico radial, as novas simulações apresentam o mesmo comportamento observado nos estudos anteriores [14]. 


\subsection{Ondas de deriva no TCABR}

Como mencionado anteriormente, a sonda de 18 pinos permite a determinação de um perfil radial para o potencial do plasma na região de borda. Para dois disparos um com eletrodo polarizado em $250 \mathrm{~V}$ e outro sem polarização temos o seguinte perfil de campo elétrico radial mostrado na Figura 3.4:

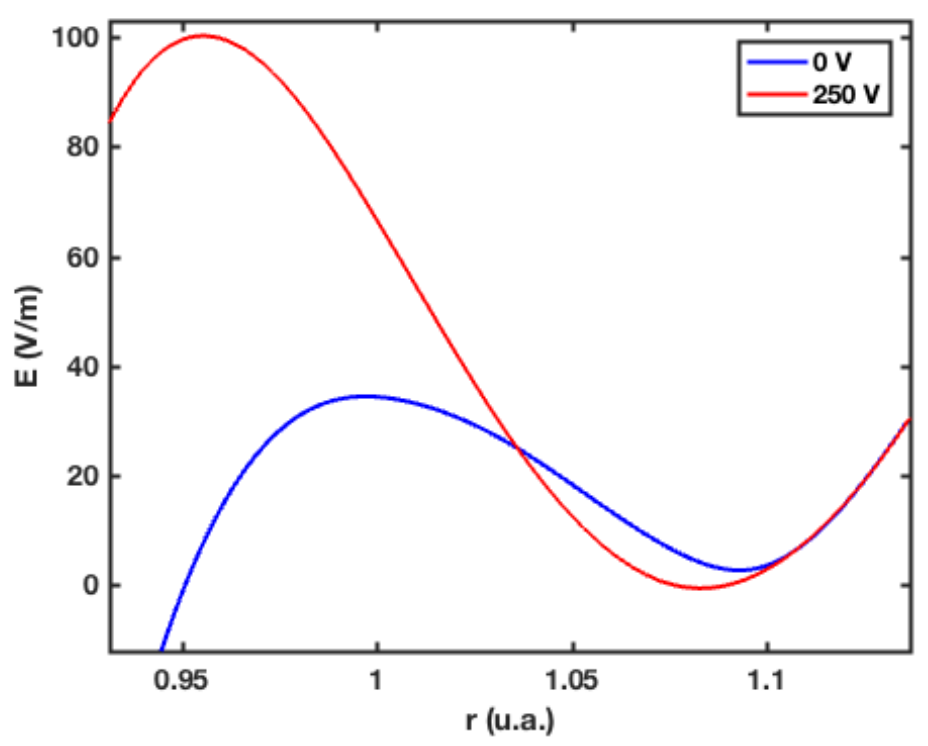

Figura 3.4: Perfil de campo elétrico para disparo sem eletrodo polarizado (azul) e polarizado em 250V (vermelho).

Estes perfies apresentam uma grande variação na direção radial na região de borda do TCABR, tal que utilizar um valor fixo para essa região não é representativo da dinâmica dessa região.

Assim foram utilizados os perfies experimentais de $E_{r}$ como uma função dependente do raio (aqui $18 \mathrm{~cm}$ foi normalizado) nas rotinas.

Sem a polarização do eletrodo, para uma onda, obtemos a Figura 3.5a. Essa apresenta um conjunto misto de órbitas abertas e fechadas na região de borda ( $r \approx 1$, portanto com a introdução de uma segunda onda, Figura 3.5b, aparece um grande transporte radial. 


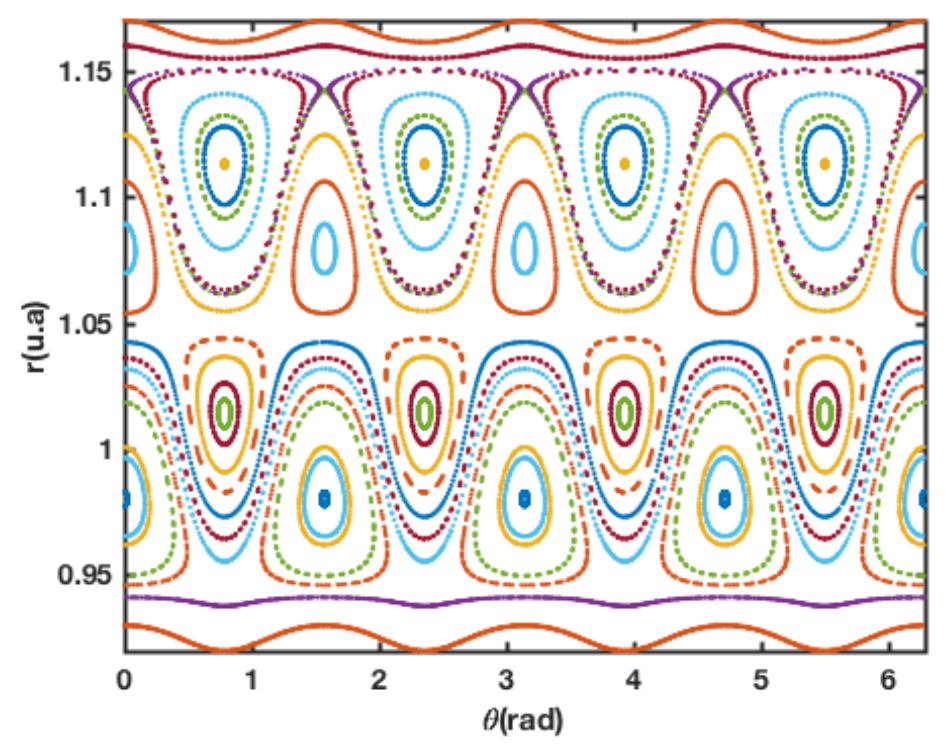

(a) Uma onda

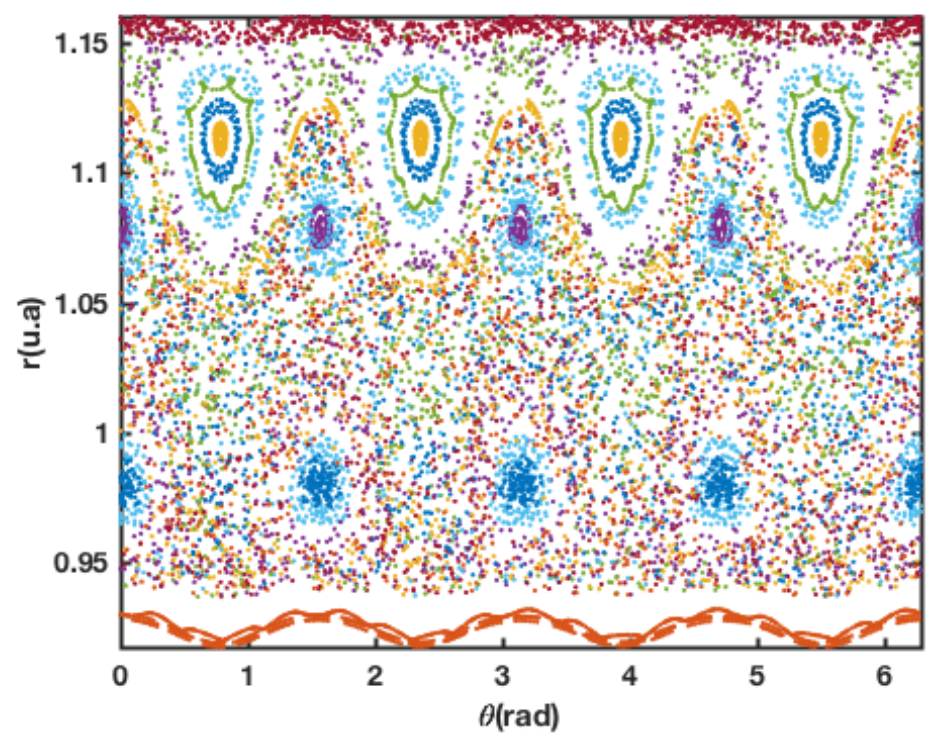

(b) Duas ondas

Figura 3.5: Mapa estroboscópico para perfil de $E_{r}$ sem polarização de eletrodo

Com o eletrodo polarizado há uma brusca mudança no campo elétrico. Essa mudança faz com que as órbitas na região de borda se tornem abertas, enquanto que as órbitas fechadas ficam contidas na região interna do plasma $(\stackrel{\circ}{<} 0.95 \sim 17 \mathrm{~cm}$ como apresentado na Figura 3.6a. Na presença de uma segunda onda as órbitas fechadas, que propagam radialmente, ficam contidas por essa barreira formada pelas órbitas abertas, como apresentado na Figura 3.6b. 


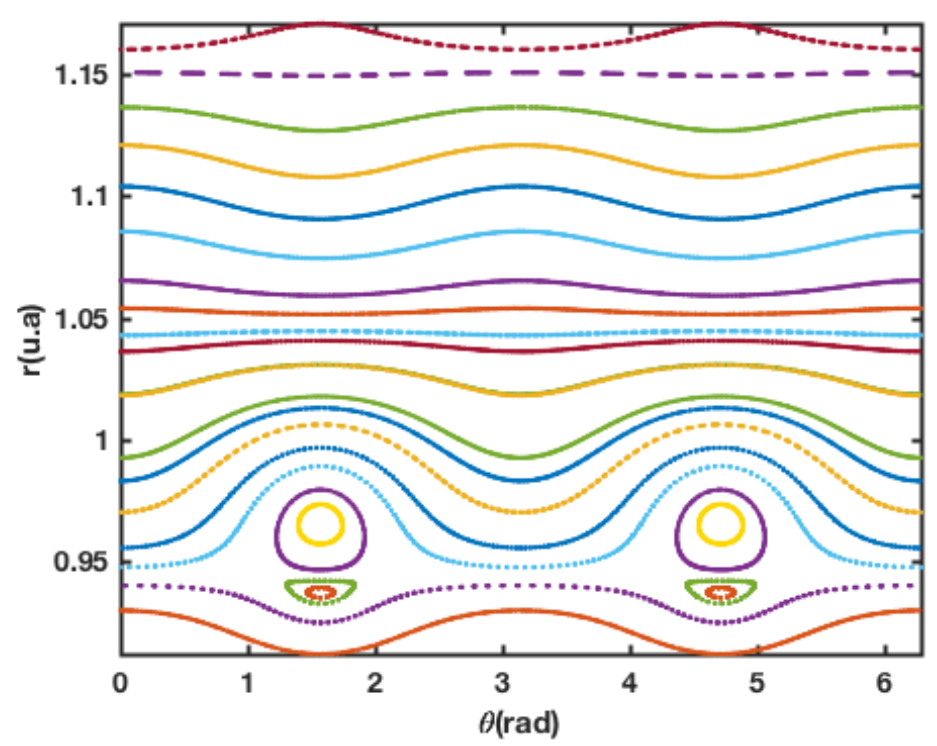

(a) Uma onda

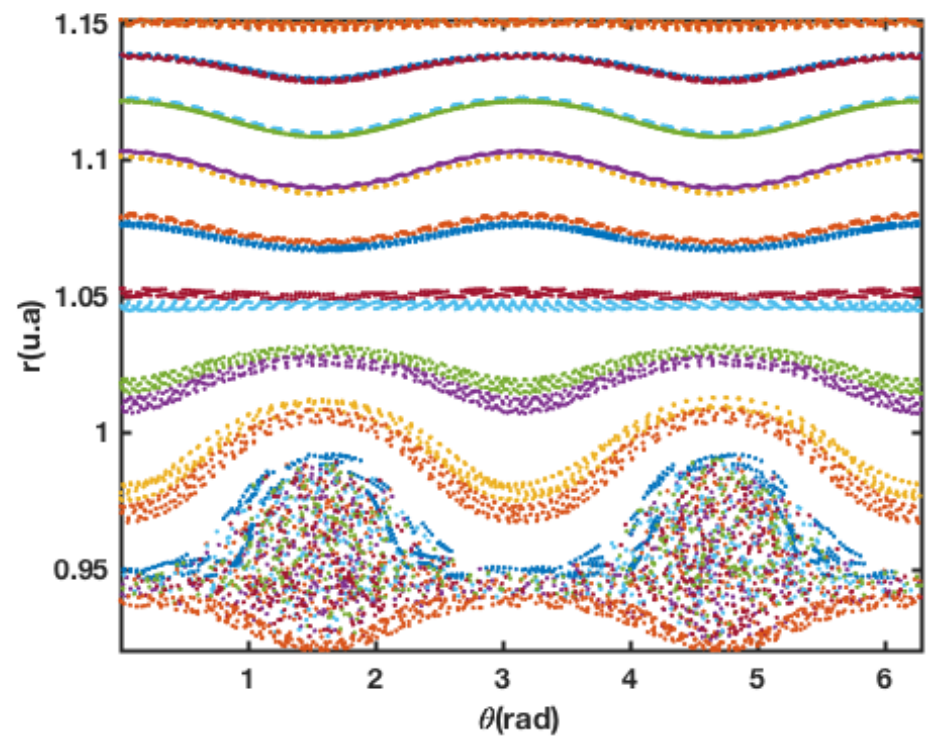

(b) Duas ondas

Figura 3.6: Mapa estroboscópico para perfil de $E_{r}$ com eletrodo polarizado em 250V

Portanto foi observado que a polarização do eletrodo transforma a borda, região propicia ao transporte radial, em uma região de barreira, dificultando assim o transporte radial e consequente perda de partículas.

Esse resultado obtido com os perfies radiais do campo elétrico, reforçam os resultados obtidos anteriormente por perfies aproximados[14]. 


\section{Capítulo 4}

\section{Bursts no Tokamak TCABR}

Um dos grandes problemas do transporte de partículas para fora do plasma é a consequente degradação do confinamento e a introdução de impurezas no plasma, estas últimas que possuem um papel negativo na condições ideais do plasma. O transporte de partículas na borda e região externa do plasma, chamada de Scrape-off-Layer (SOL), é dominado por estruturas coerentes de grande densidade (picos vários desvios-padrões acima da densidade média), chamadas aqui de "bursts" [12, 26, 25, 24].

A região de borda do plasma apresenta flutuações de densidade intermitentes de alta amplitude, estas flutuações tem sido relacionadas a existência de estruturas coerentes de alta densidade e temperatura, chamadas de "bursts". Estas estruturas devido a sua natureza são responsáveis por uma parte importante do transporte de partículas e energia para fora do plasma e podem acabar danificando as paredes da câmara de confinamento ao se chocarem com esta. Assim o estudo dessas estruturas tem ganhado grande atenção nas últimas décadas.

\subsection{O processo de propagação convectivo dos bursts}

Acreditava-se que o transporte de partículas para fora do plasma seguia um processo difusivo, porém os experimentos tem mostrado níveis de transporte incompatíveis com os esperados por processos difusivos [11]. Além disso, medidas de turbulência na borda apresentam comportamento intermitente e estruturas de densidade e temperatura muito superiores ao fundo, como pode ser visto nas Figuras 4.1 4.2 e 4.3. 


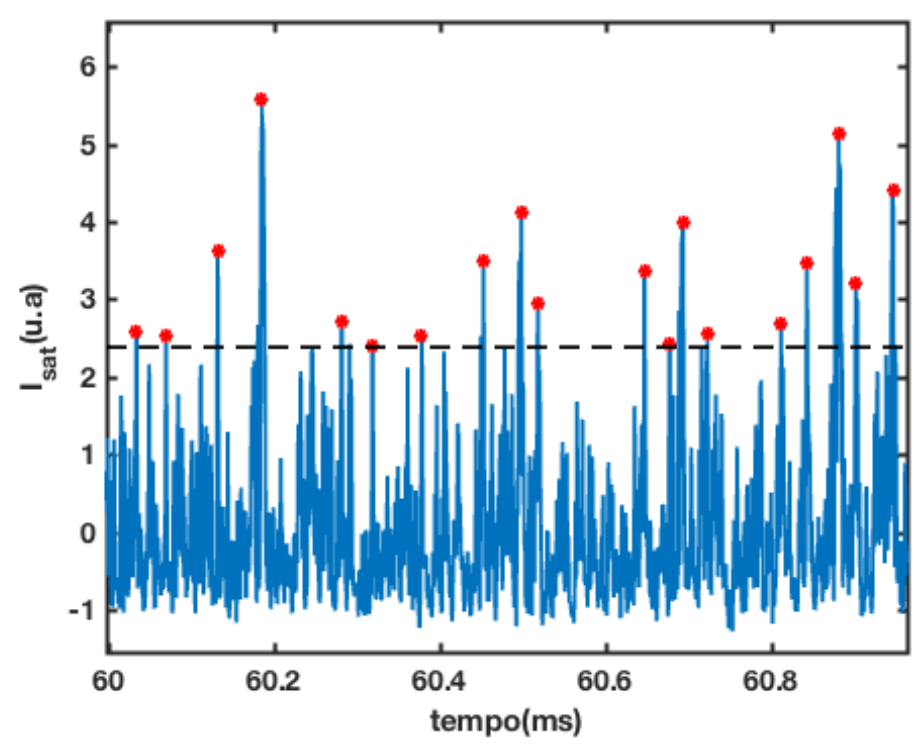

Figura 4.1: Exemplo de sinal normalizado de corrente de saturação iônica (proporcional às flutuações de densidade local do plasma) mostrando a presença de picos mais de 2.5 desvios-padrões acima do valor médio, os quais são identificados como bursts.

A figura 4.1 apresenta a corrente de saturação de iônica na região de borda do TCABR, onde os eventos de alta densidade detectados nesta amostra de $1 \mathrm{~ms}$ foram marcados em vermelho. A alta assimetria do sinal é observada no histograma 4.2.

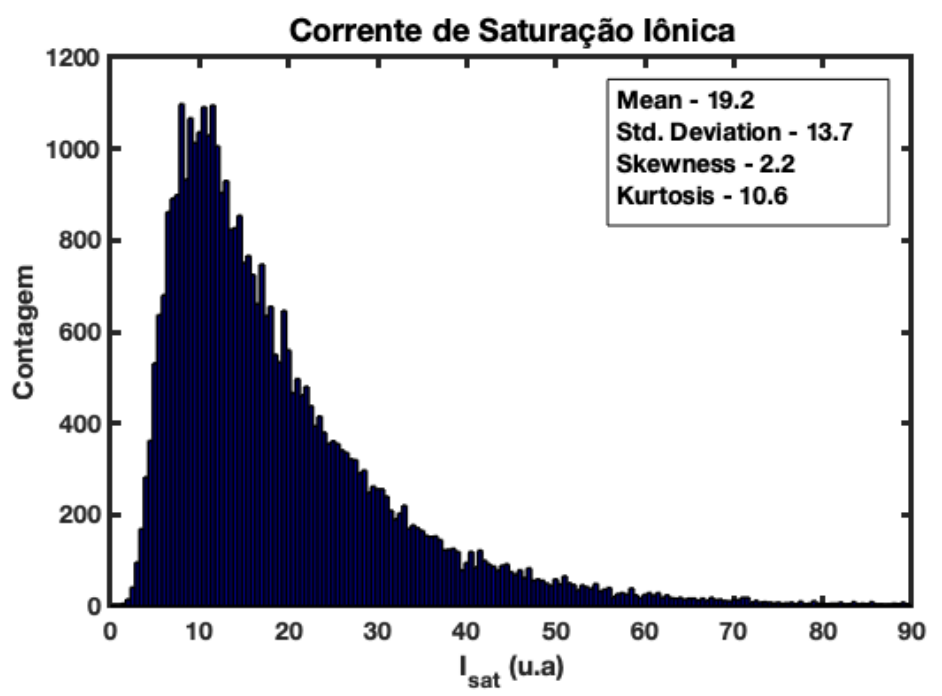

Figura 4.2: Histograma da corrente de saturação iônica mostrando uma assimetria positiva bastante acentuada.

Este fenômeno foi observado em diversas máquinas (Figura 4.3), demonstrando a sua universalidade e relevância para o transporte da região de borda.

Estes fenômenos têm sido atribuídos à presença de estruturas coerentes tri-dimensionais que se formam na região de borda e se propagam radialmente [24]. Sua formação tem sido 


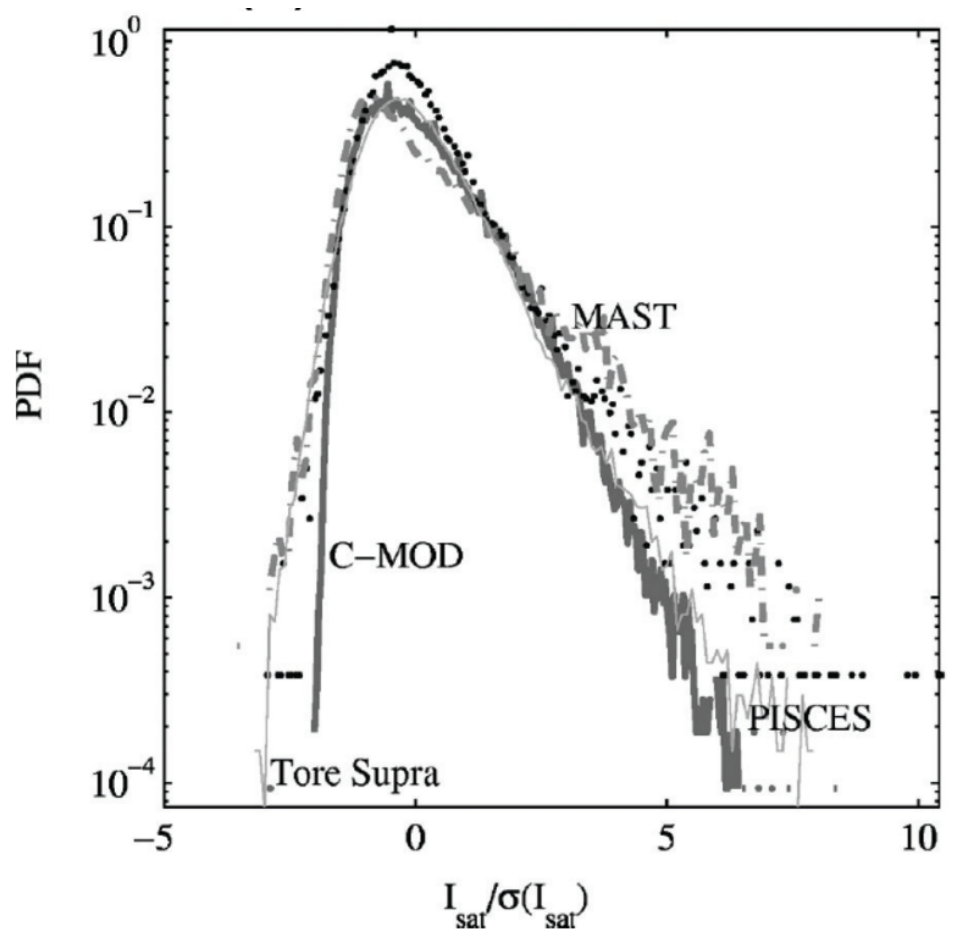

Figura 4.3: Histogramas de corrente de saturação iônica normalizada obtidos em diversas máquinas de confinamento magnético de plasmas - Tore Supra, Alcator C-MOD e MAST são tokamaks, e PISCES é um dispositivo linear. Figura extraída da referência [26]

relacionada a gradientes de densidade provenientes de processos turbulentos, que expostos a forças que geram a separação local de partículas, acabam criando uma estrutura dipolar que então propaga com velocidade de deriva $E \times B$, como ilustrado na Figura 4.4. 


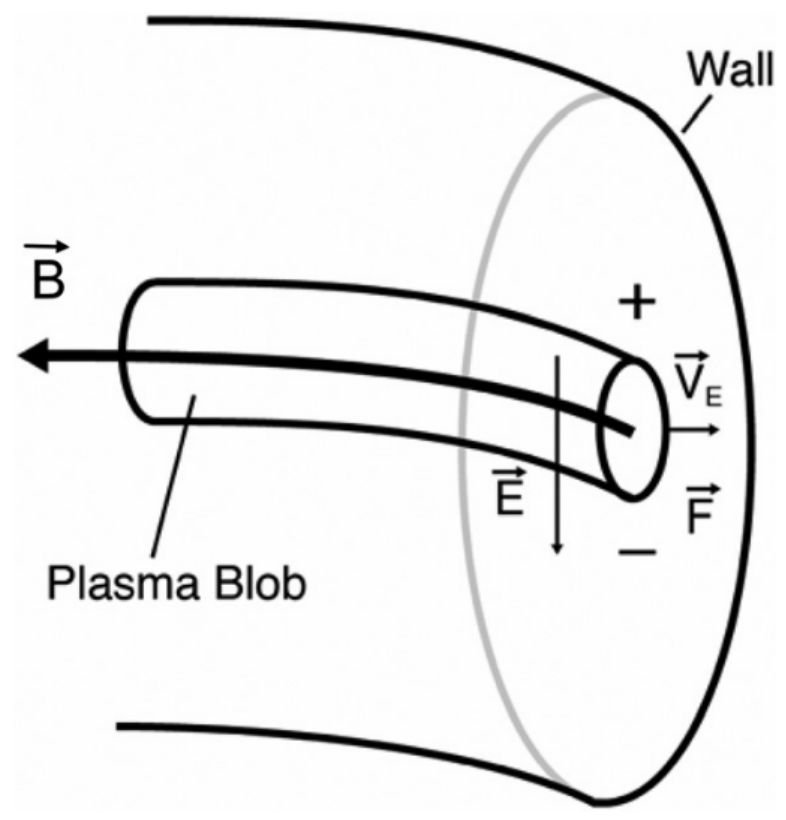

Figura 4.4: Representação esquemática do processo de formação dos bursts por meio da deriva provocada pela curvatura e gradiente do campo magnético. Figura extraída da referência [24].

Essas estruturas fazem com que a distribuição de probabilidade dos sinais (PDF) nesta região apresente elevados valores de assimetria (skewness) e curtose (kurtosis) características deste fenômeno intermitente, como pode ser visto nas Figuras 4.2 e 4.3. Além disso as estruturas possuem uma forma de pulso característica ao serem detectadas por sondas eletrostáticas.

Estas figuras são criadas a partir da média dos picos detectados, a chamada média condicional, como mostrado na Figura 4.5. Além disso, como mostrado na Figura 4.10, a forma típica das estruturas depende da posição radial, sendo que o tempo para a densidade retornar ao valor que tinha antes da passagem da estrutura (tempos de atraso relativo, $\Delta$, maiores que zero) aumenta com o aumento da posição radial, ao passo que o tempo para a densidade subir até atingir o pico é praticamente a mesma em todas as posições radiais consideradas. Através desse tipo de análise é possível estudar as velocidades de propagação das estruturas ao se comparar o atraso entre as medições em pinos distintos das sondas.

Exemplos de atrasos entre sinais medidos em sondas distintas podem ser vistos nas Figuras 4.7 a 4.9. 


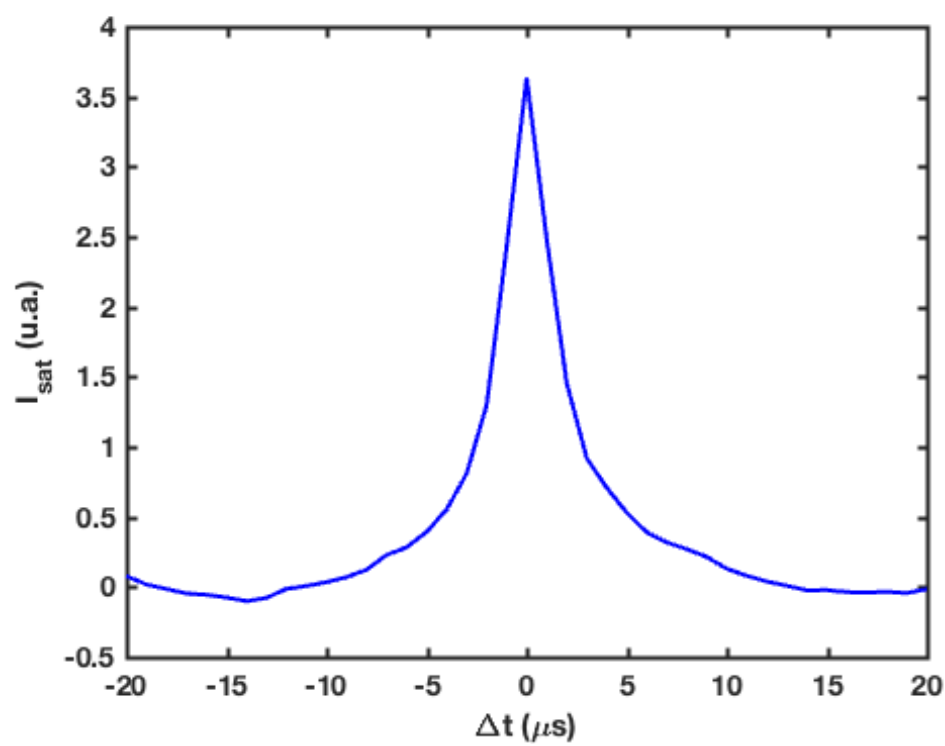

Figura 4.5: Perfil temporal típico dos bursts observados no tokamak TCABR, obtido pelo uso da técnica de análise condicional. A escala horizontal representa a diferença de tempo em relação ao momento em que os picos identificados como bursts são detectados na sonda.

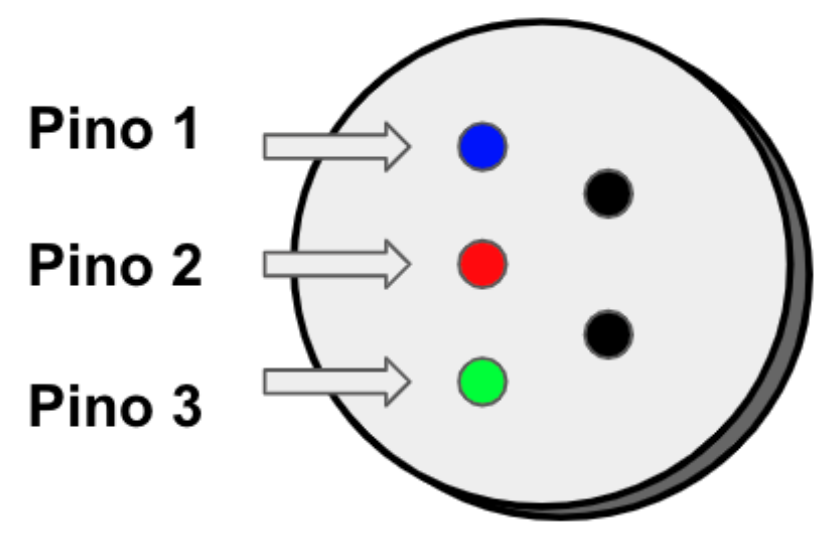

Figura 4.6: Esquema de arranjo dos pinos, cores referentes aos sinais a seguir.

Quando detectamos os burts (pino de referência) no pino superior, podemos observar que em média os bursts tem uma trajetória 1-2-3 (Figura 4.7), pois são detectados primeiro no pino 1 , após um delta de tempo no pino 2 e por fim no pino 3 , indicando um movimento na direção em que os pinos estão ordenados. 


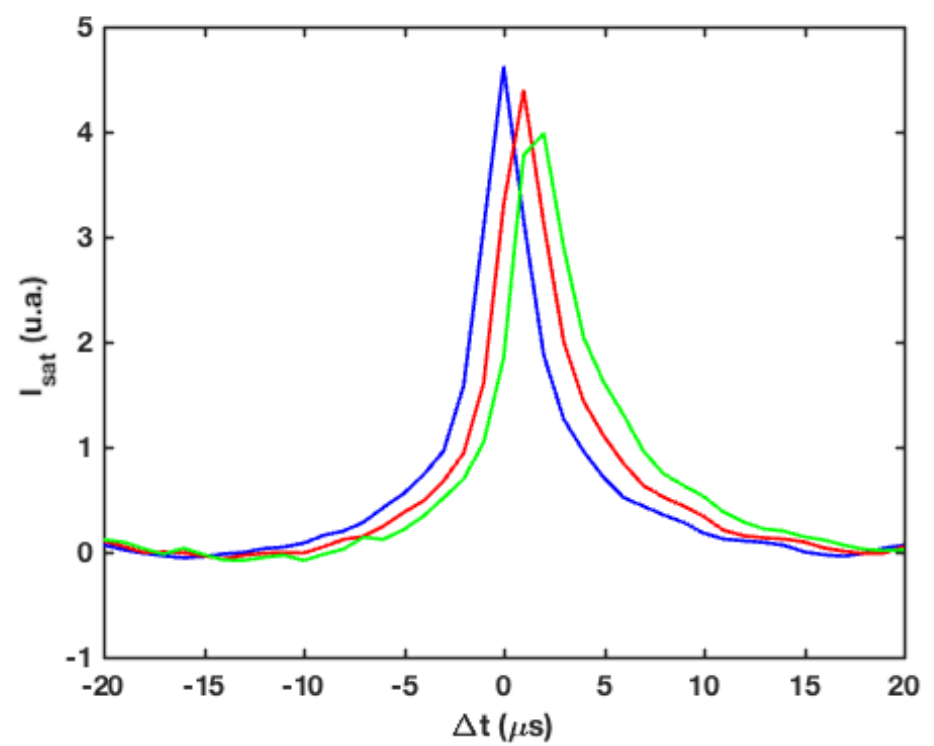

Figura 4.7: Média condicional cruzada dos bursts detectados nas três sondas dispostas verticalmente (poloidalmente) na sonda de cinco pinos com a referência para identificação dos tempos condicionados definida pelo pino superior da sonda (curva em azul). As curvas em vermelho e verde correspondem, respectivamente, aos sinais medidos nos pinos localizados $0.5 \mathrm{~cm}$ e $1.0 \mathrm{~cm}$ abaixo do pino de referência.

O mesmo movimento médio é observado na figura 4.8, ao selecionar como referencia o pino central vemos que o bursts foram detectados primeiramente no pino 1 ( $\Delta t$ negativo) e por último no pino 3. 


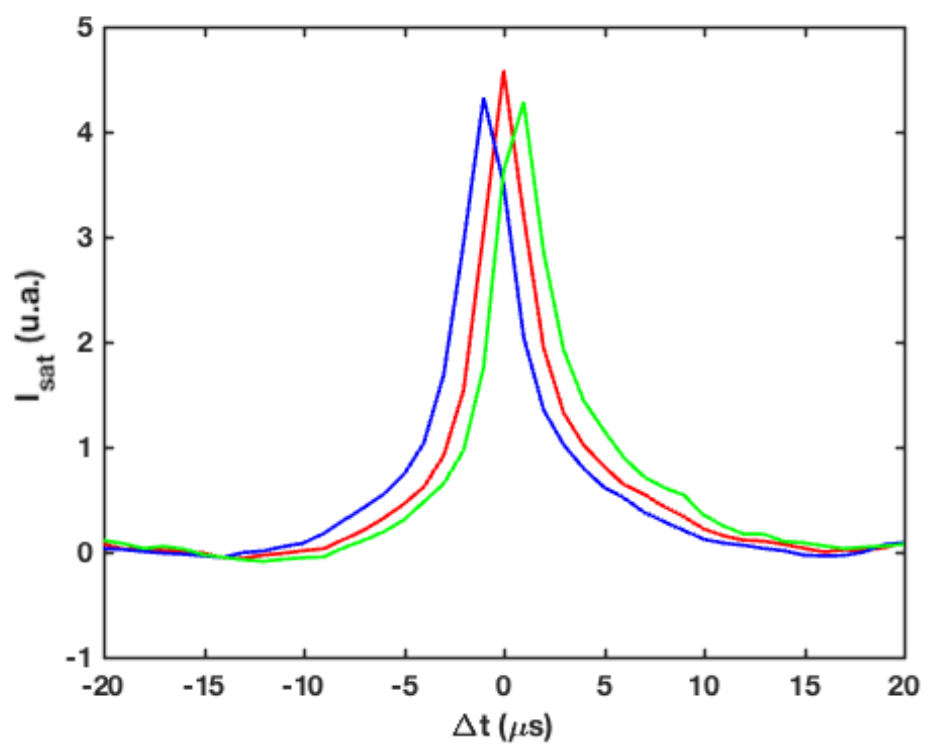

Figura 4.8: Similar à Figura 4.7, porém, para o caso em que o pino de referência para a média condicional é o pino central da sonda (curva em vermelho). O sinal azul corresponde ao pino superior $(0.5 \mathrm{~cm}$ acima do pino de referência) e a curva verde ao pino inferior ( $0.5 \mathrm{~cm}$ abaixo do pino de referência).

E como esperado, quando o pino de referencia é o pino 3, os deltas para os demais pinos são negativos (Figura 4.9).

Este comportamento médio, obtido através de centenas de bursts, indica uma possível dinâmica característica dos bursts. 


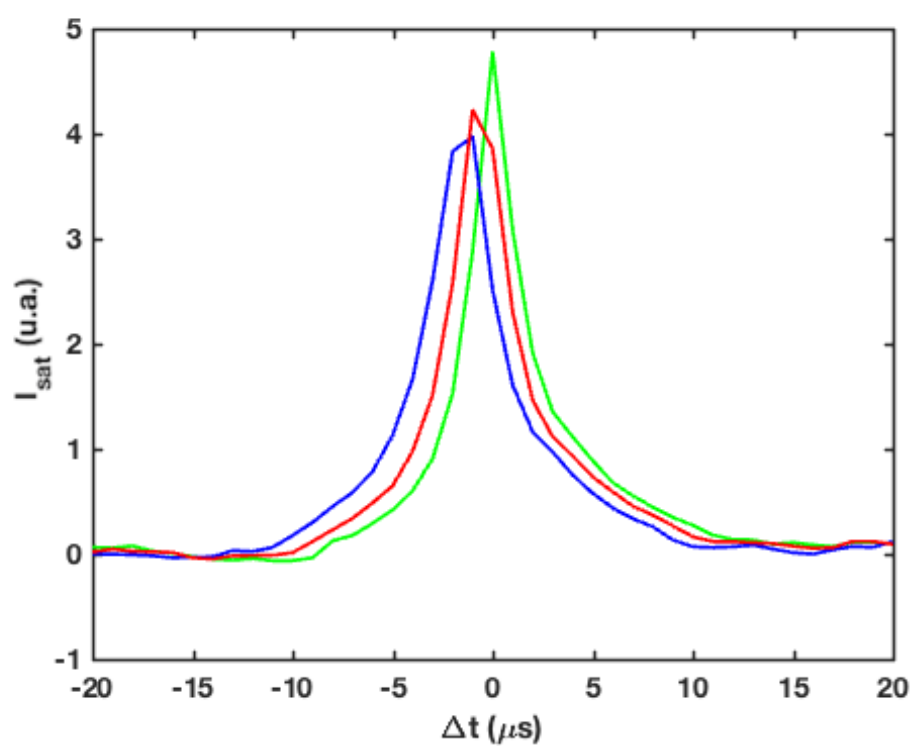

Figura 4.9: Similar às Figuras 4.7 e 4.8, porém, para o caso em que o pino de referência para a média condicional é o pino inferior da sonda (curva em verde). O sinal azul corresponde ao pino superior ( $1.0 \mathrm{~cm}$ acima do pino de referência) e a curva vermelha ao pino central da sonda $(0.5 \mathrm{~cm}$ acima do pino de referência).

Outra característica observada com o uso da sonda de 18-pinos, e portanto temos uma visão dos burts em diferentes posições radiais, é o inicio dos burts ser bem definido, representado pelo pequeno $\Delta t$ entre o inicio do burst e o pico, enquanto o fim do burst apresenta um tempo maior de queda, indicando um possível alongamento da estrutura. Esse fenomeno como pode ser visto na figura4.10 é intensificado na direção do limitador (quanto maior o r). Além da cauda também é possível notar a diminuição de amplitude do burst. 


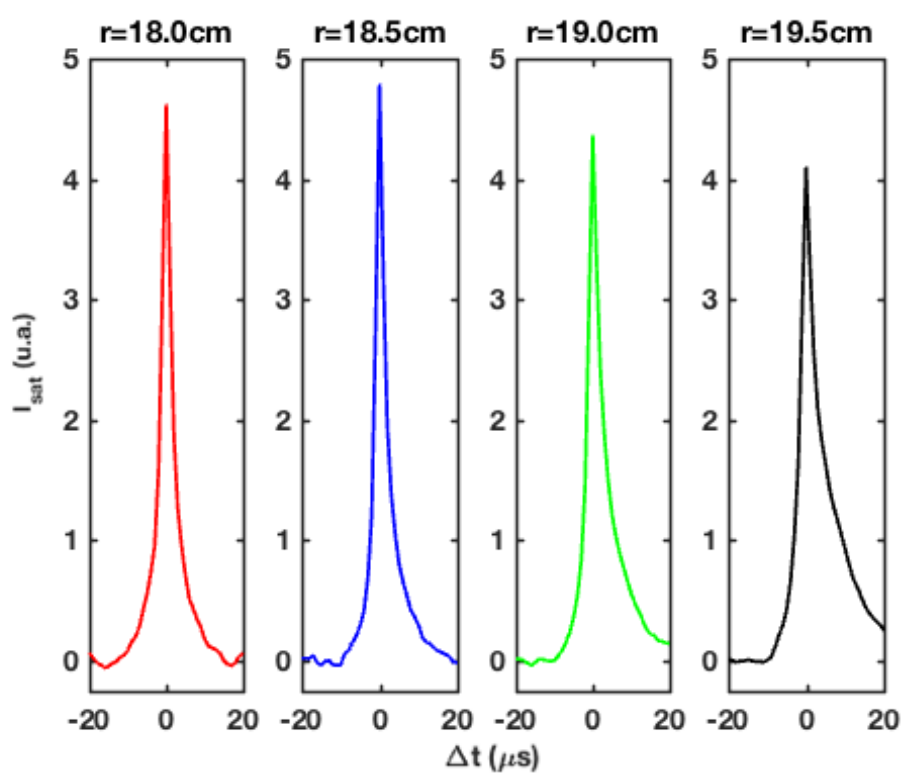

Figura 4.10: Formato condicional dos bursts em diferentes posições radiais na região de borda e sombra do limitador. A escala vertical é a corrente de saturação normalizada e a escala horizontal é o tempo em relação ao momento que os picos identificados como bursts são detectados no sinal de $I_{\text {Sat }}$.

$\mathrm{O}$ arranjo poloidal de sondas nos permite calcular a velocidade radial de $E \times B$ das estruturas $\left(v_{r}=\delta \phi / d \theta\right)$, através da medida de potencial.

Estudando a relação entre a amplitude das estruturas (proporcional ao tamanho) e a sua velocidade de propagação radial obtemos a figura 4.11.

Como pode ser visto na Figura 4.11, não há evidências de que a velocidade de propagação radial dependa da amplitude da perturbação de densidade associada às estruturas.

Este fenômeno sugere que a simples quebra das estruturas maiores em outras menores por conta do cisalhamento da velocidade poloidal pode não ser suficiente para explicar a melhoria de confinamento observada na presença do eletrodo. 


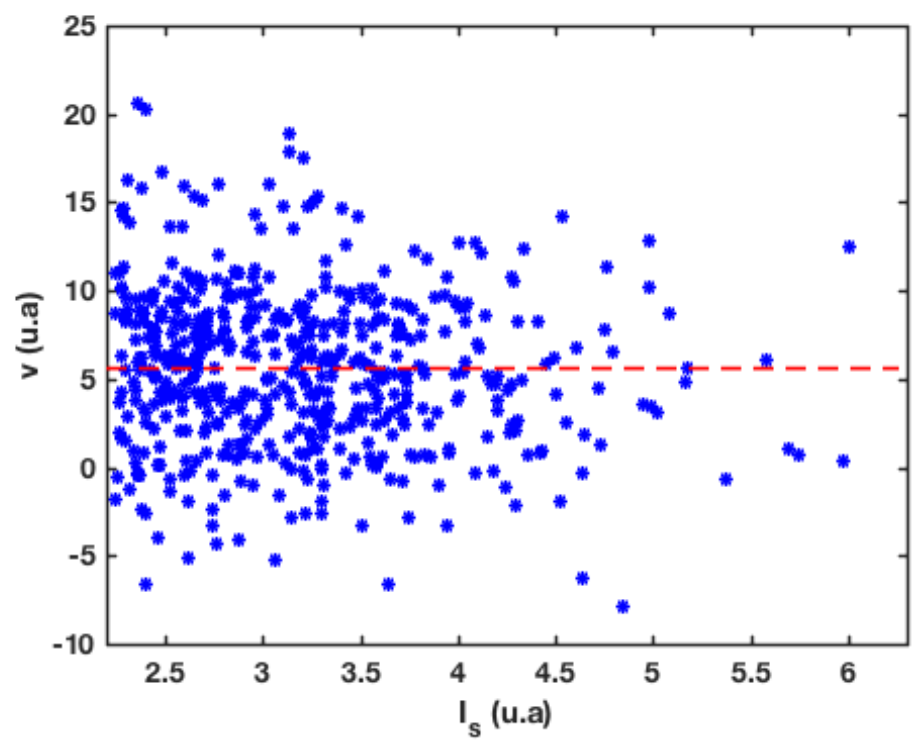

Figura 4.11: Relação entre a velocidade radial $\left(v_{r}=\delta \phi / d \theta\right)$ e a amplitude da perturbação de densidade provocada pelo burst. A linha tracejada em vermelho indica o valor médio da velocidade radial de todos os bursts representados. Velocidades positivas indicam bursts se movendo para fora do plasma.

\subsection{Efeito do eletrodo sobre os Bursts}

A redução do transporte associada a polarização de eletrodo na borda do plasma, como esperado, também tem suas consequências observadas na forma tipica dos bursts, como mostrado nas Figuras 4.12 a 4.14, que mostram que o tempo de subida e de descida do pico de densidade dos bursts é significativamente reduzido durante o período em que o eletrodo é polarizado (entre 60 e $80 \mathrm{~ms}$ )." 


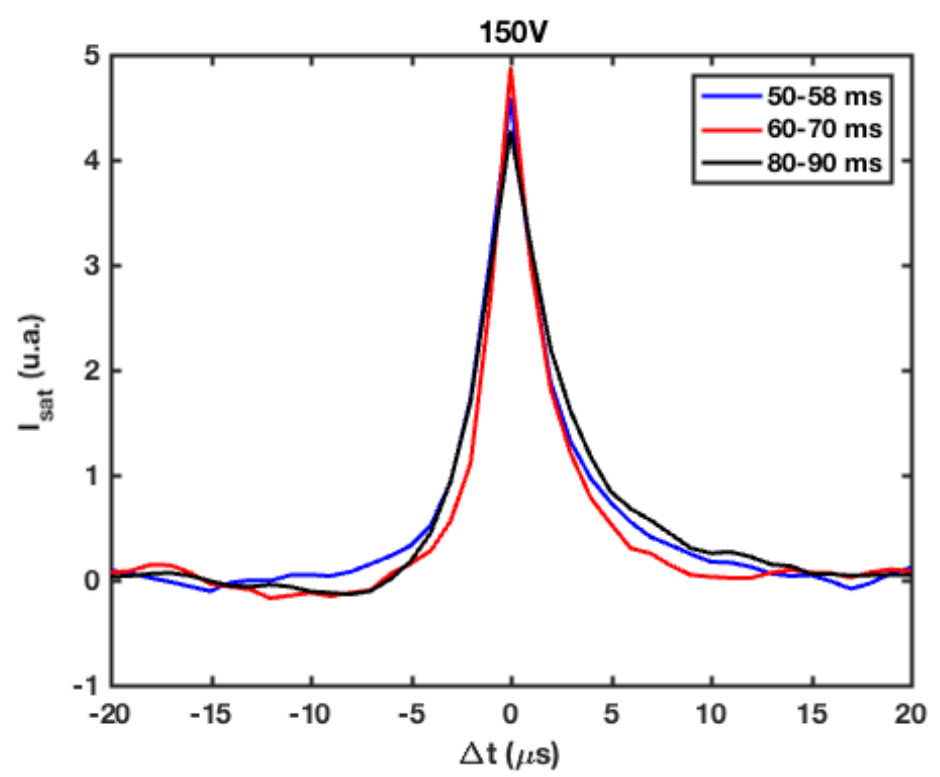

Figura 4.12: Formato condicional dos bursts para um disparo com polarização do eletrodo em $150 \mathrm{~V}$. O sinal azul corresponde ao período antes da polarização do eletrodo, o sinal vermelho ao período em que o eletrodo estava polarizado e o preto ao período pós polarização.

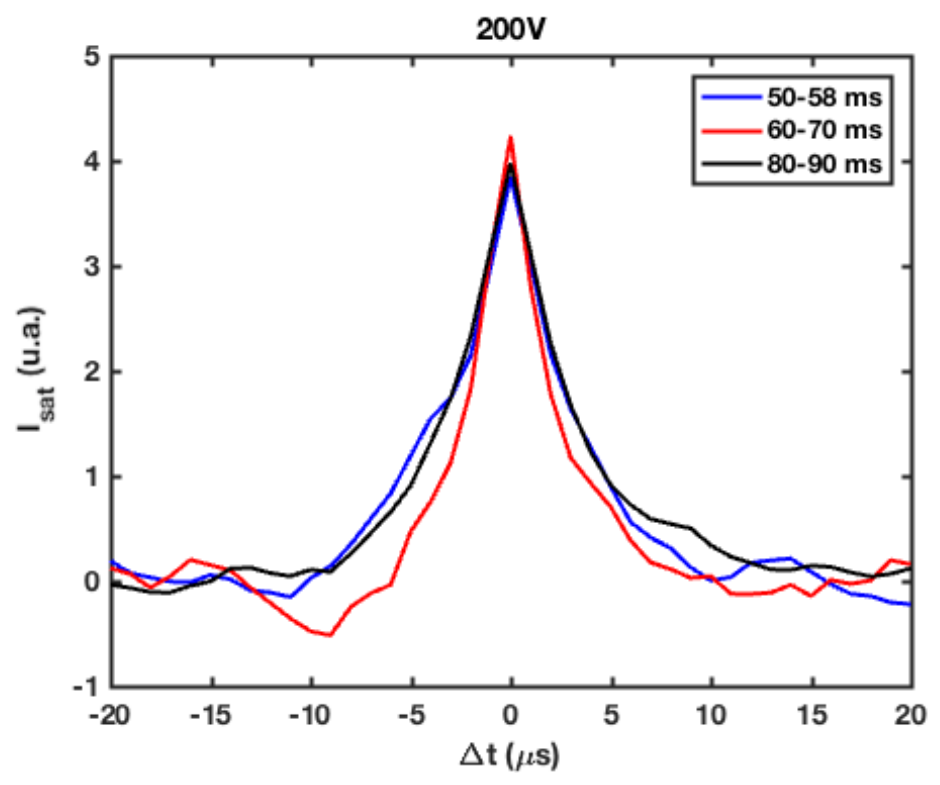

Figura 4.13: Formato condicional dos bursts para um disparo com polarização do eletrodo em $200 \mathrm{~V}$. O sinal azul corresponde ao período antes da polarização do eletrodo, o sinal vermelho ao período em que o eletrodo estava polarizado e o preto ao período pós polarização. 


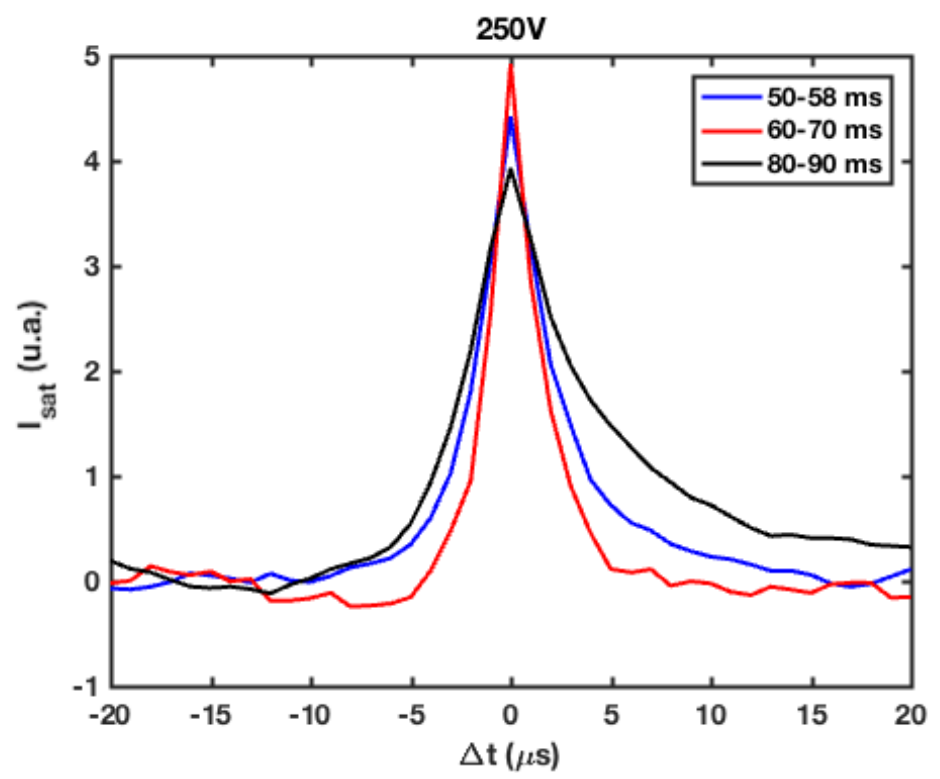

Figura 4.14: Formato condicional dos bursts para um disparo com polarização do eletrodo em $250 \mathrm{~V}$. O sinal azul corresponde ao período antes da polarização do eletrodo, o sinal vermelho ao período em que o eletrodo estava polarizado e o preto ao período pós polarização.

É possível identificar a relação entre a intensidade do estreitamento do burst, representando uma estrutura menos alongada, e a tensão aplicada.

Esta mudança no formato dos picos é observada principalmente na região interna à coluna de plasma $(r<18 \mathrm{~cm})$ e se torna menos relevante à sombra do limitador como mostrado na Figura 4.15, indicando o possível papel da barreira na mudança das estruturas.

Apesar da mudança do formato dos burst e diminuição da amplitude observados durante a aplicação do eletrodo é importante notar que estas estrutura continuam presentes na borda do plasma, e recuperam suas características originais assim que o eletrodo é despolarizado, como pode ser observados em todos os disparos. 

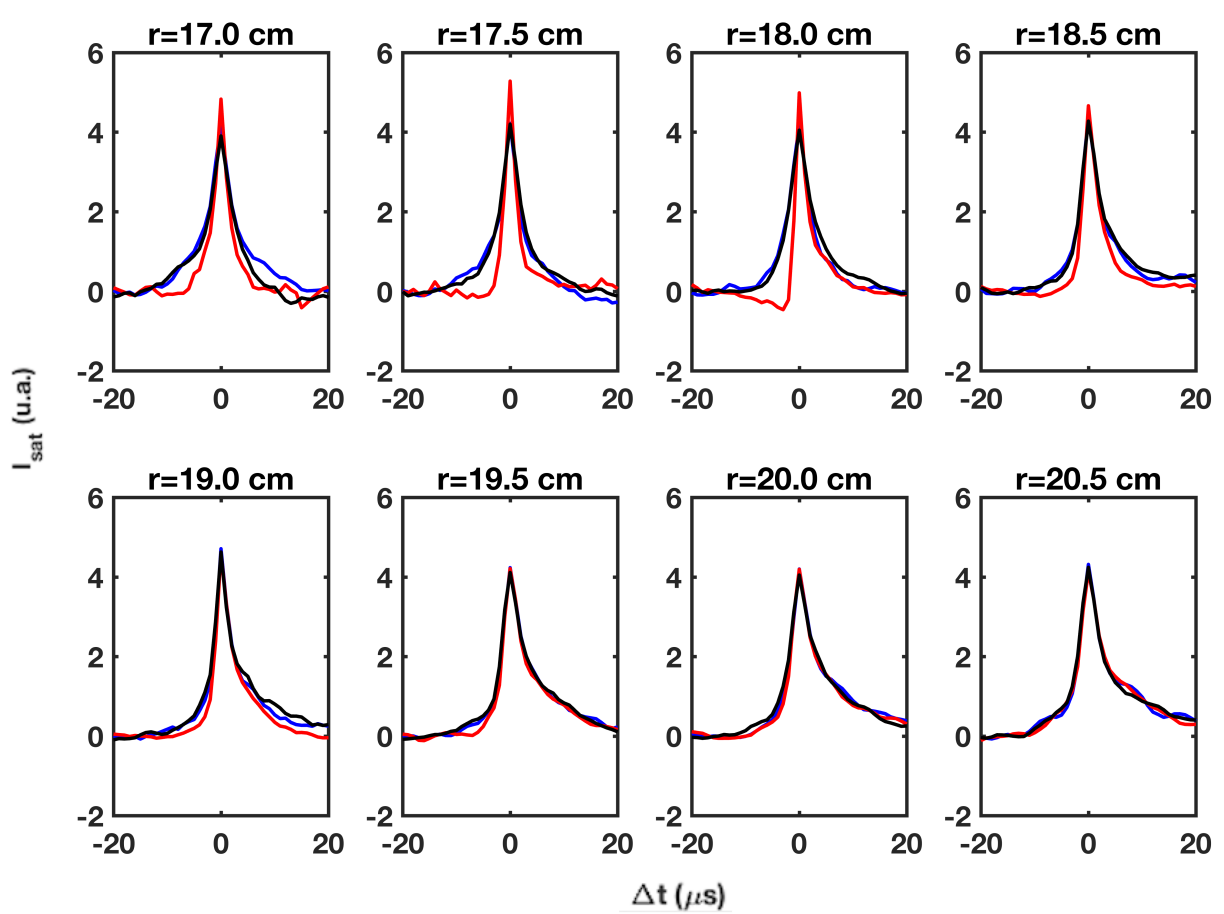

Figura 4.15: Formato condicional dos bursts em diferentes posições radias para um disparo com polarização do eletrodo em $250 \mathrm{~V}$. O sinal azul corresponde ao período antes da polarização do eletrodo, o sinal vermelho ao período em que o eletrodo estava polarizado e o preto ao período pós polarização. 


\section{Capítulo 5}

\section{Conclusão}

Considera-se que um dos mecanismos fundamentais da melhora de confinamento observada na transição do modo $L$ para o modo $H$, seja a redução do transporte turbulento pelo cisalhamento do fluxo $E \times B$, causando a quebra de estruturas coerentes, diminuindo o tamanho radial dessas estruturas e portanto diminuindo o transporte decorrente dessas estruturas de alta densidade [11, 22, 28, 17, 27].

Porém, não há um modelo de primeiros princípios que descreva a redução do transporte e pouco se é conhecido sobre o transporte na borda do plasma. Dado esse cenário, o uso de simulações computacionais de dinâmicas simplificadas é um importante aliado para identificar os mecanismos essenciais do fenômeno. Um destes modelos foi estudado no capitulo 3, abordando a dinâmica caótica e o consequente transporte radial proveniente da deriva $E \times B$ de partículas na presença de ondas de deriva. Como observado em estudos anteriores [14] a diferença de fase entre a velocidade de fase das ondas de deriva e a velocidade do fluxo $E \times B$ gerado pelo capo elétrico radial, está associada ao surgimento de orbitas propicias ao transporte radial ou de orbitas abertas que formam barreiras a esse transporte.

Neste trabalho, foram utilizados valores atualizados de perfis radiais dos parâmetros do plasma no TCABR, observando o surgimento de uma barreira na região esperada, quando se considera o perfil radial do campo elétrico proveniente de disparos com a polarização do eletrodo. As simulações feitas para as condições do plasma em descargas não perturbadas no TCABR não exibem barreiras de transporte, reforçando a compatibilidade do modelo com observações experimentais.

Apesar de apresentar a formação da barreira de transporte, o modelo baseado na dinâmica caótica das partículas na presença de ondas de deriva representa apenas o transporte difusivo e não considera o transporte convectivo associado as estruturas coerentes.

A dinâmica dessas estruturas coerentes observadas na região de borda do TCABR foram investigadas no capitulo 4 a partir das medidas de sondas eletrostáticas inseridas na 
região periférica do TCABR. Os resultados desta análise mostram que as estruturas possuem um perfil temporal característico que varia levemente com a posição radial, porém sua velocidade radial não apresenta dependência clara com a intensidade da perturbação de densidade associada à essas estruturas. Vale a pena destacar que este comportamento inesperado também foi observado em estudos recentes do grupo em descargas de plasma no Texas Helimak [29], e é um ponto relevante a ser investigado em estudos futuros.

Como esperado, observamos que as estruturam sofrem alterações durante a polarização do eletrodo, tendo como efeitos principais a diminuição da intensidade e o estreitamento do perfil temporal da estrutura, com uma redução significativa dos tempos de subida e decida da intensidade local. Observamos ainda que a intensidade da mudança aumenta com a tensão aplicada ao eletrodo.

Apesar das estruturas sofrerem alterações pela polarização do eletrodo que são consistentes com o surgimento de uma barreira de transporte, e os níveis de transporte observados diminuírem como consequência da polarização do eletrodo, a falta de dependência entre a velocidade radial de propagação das estruturas e sua intensidade pode indicar que apenas o mecanismo de quebra de estruturas coerentes não seja suficiente para explicar a redução da perda de partículas e energia observadas experimentalmente na região de borda do plasma quando o eletrodo é polarizado. 


\section{Referências}

[1] Hannah Ritchie e Max Roser. Fossilfuels. URL: https : / / ourwor ldindata . org/fossil-fuels.

[2] British Petroleum. Statistical Review of World Energy. URL: https : / / www . bp . com/en/global / corporate/energy-economics/statisticalreview-of-world-energy.html.

[3] Hannah Ritchie. How long before we run out of fossil fuels? 2017. URL: https: / / ourworldindata . org / how-long-before-we-run-out - of fossil-fuels.

[4] Bart Verberck e Andrea Taroni. "Nuclear fusion". Em: Nature Physics 12 (mai. de 2016), p. 383.

[5] Elizabeth Gibney. "Fuel for world's largest fusion reactor ITER is set for test run". Em: Nature 591 (mar. de 2021), p. 15. DoI: https : / / doi . org/10.1038/ d41586-021-00408-1.

[6] Francis F. Chen. Introduction to Plasma Physics and Controlled Fusion. Vol. 3. Springer International Publishing, 2016. ISBN: 978-3-319-22309-4.

[7] George Patrick Lasche. "Magnetic confinement of fusion plasmas". Em: The Physics Teacher 19.4 (1981), pp. 234-244. DOI: $10.1119 / 1.2340764$.

[8] J. Wesson e D.J. Campbell. Tokamaks. International Series of Monogr. OUP Oxford, 2011. ISBN: 9780199592234.

[9] F. Wagner. “A quarter-century of H-mode studies”. Em: Plasma Physics and Controlled Fusion 49.12 B (2007).

[10] B Lipschultz. "Overview of the JET results in support to ITER". Em: Nuclear Fusion 10 (2017).

[11] “Chapter 2: Plasma confinement and transport". Em: Nuclear Fusion 39.12 (1999), pp. 2175-2249. ISSN: 0029-5515. DOI: $10.1088 / 0029-5515 / 39 / 12 / 302$. 
[12] G. Y. Antar et al. "Turbulence intermittency and burst properties in tokamak scrapeoff layer”. Em: Physics of Plasmas 8.5 I (2001), pp. 1612-1624. ISSN: 1070664X. DOI: $10.1063 / 1.1363663$.

[13] "Plasma confinement using biased electrode in the TCABR tokamak". Em: $\mathrm{Nu}$ clear Fusion 45.8 (2005), pp. 796-803. ISSN: 00295515. DOI: 10 . $1088 / 0029$ $5515 / 45 / 8 / 005$.

[14] Francisco Alberto Marcus. "Transporte de partículas induzido por ondas de deriva". Tese de dout. 2007.

[15] F. A. Marcus et al. "Reduction of chaotic particle transport driven by drift waves in sheared flows". Em: Physics of Plasmas 15.11 (2008). ISSN: 1070664X. DOI: $10.1063 / 1.3009532$.

[16] I. L. Caldas et al. "Dynamical Effects in Confined Plasma Turbulence". Em: Brazilian Journal of Physics 44.6 (2014), pp. 903-913. ISSN: 16784448. DOI: 10 . $1007 / \mathrm{s} 13538-014-0259-\mathrm{x}$.

[17] Gustavo Guedes Grenfell. "Estudo de Efeitos da Polarização Eletrostática Periférica no Tokamak TCABR". Tese de dout. 2016.

[18] G. G. Grenfell et al. "H-mode access and the role of spectral shift with electrode biasing in the TCABR tokamak". Em: Physics of Plasmas 25.7 (2018). ISSN: 10897674. DOI: $10.1063 / 1.5029561$.

[19] Wilson Andres Hernandez Baquero. "Turbulent temperature fluctuations in TCABR tokamak". Em: (2017).

[20] I. H. Hutchinson. Principles of Plasma Diagnostics. 2002. ISBN: 9780521803892. DOI: $10.1017 / \mathrm{cbo} 9780511613630$.

[21] J. D. Callen. "Transport processes in magnetically confined plasmas". Em: Physics of Fluids B 4.7 (1992), pp. 2142-2154.

[22] Radu Balescu. Aspects of Anomalous Transport in Plasmas. Vol. 18. 2005. ISBN: 978-0-7503-1030-7. DOI: $10.1201 / 9781420034684$.

[23] W. Horton. "Drift waves and transport". Em: Reviews of Modern Physics 71.3 (1999), pp. 735-778. ISSN: 0034-6861. DOI: 10 . 1103 / RevModPhys . 71 . 735.

[24] D. A. D’Ippolito, J. R. Myra e S. J. Zweben. “Convective transport by intermittent blob-filaments: Comparison of theory and experiment”. Em: Physics of Plasmas 18.6 (2011). ISSN: 1070664X. DOI: $10.1063 / 1.3594609$. 
[25] O. E. GARCIA. "Blob Transport in the Plasma Edge: a Review". Em: Plasma and Fusion Research 4 (2009), pp. 019-019. ISSN: 1880-6821. DOI: 10 . 1585 /pfr. 4.019. URL: http: / / joi.jlc.jst.go.jp/JST. JSTAGE/pfr/ 4 . 019 ? from=CrossRef.

[26] G Y Antar. "On the origin of "intermittency" in the scrape-off layer of linear magnetic confinement devices”. Em: Physics of Plasmas 10.9 (2003), pp. 3629-3634. DOI: 10 .1063/1.1599855. URL: https : / / doi .org/10 .1063/1. 1599855.

[27] "Influence of sheared poloidal rotation on edge turbulence". Em: Physics of Fluids B 2.1 (1990), pp. 1-4. ISSN: 08998221. DOI: $10.1063 / 1.859529$.

[28] Z Lin et al. "Turbulent Transport Reduction by Zonal Flow: Massively Parallel Simulations”. Em: Science 281.SEPTEMBER (1998), p. 1835.

[29] Théo L. Meireles. Burst size and velocity in Texas Helimak. 28 SIICUSP (28th USP International Symposium of Undergratuate Research). 2020.

[30] J Bittencourt. Fundamentals of Plasma Physics. Vol. 3. Jan. de 2004. ISBN: 978-0387-20975-3. DOI: 10 .1007/978-1-4757-4030-1.

[31] Dennis Lozano Toufen. "Controle da turbulência em plasmas”. Tese de dout. 2012.

[32] C. Hidalgo. "On the nature of transport in fusion plasmas". Em: Astrophysics and Space Science 292.1-4 (2004), pp. 681-690. ISSN: 0004640X. DOI: 10 . 1023 /B : ASTR. 0000045075.06166 .8 a.

[33] V. Naulin. "Turbulent transport and the plasma edge". Em: Journal of Nuclear Materials 363-365.1-3 (2007), pp. 24-31. ISSN: 00223115. DOI: $10.1016 / \mathrm{j}$. jnucmat.2006.12.058. 